Envel opes of I egendre curves i $n$ the uni $t$ spherical bundle over the unit sphere

\begin{tabular}{|l|l|}
\hline 著者 & $\begin{array}{l}\text { LI Yanl i n, PEI Donghe, TAKAHASH Nasat ond, YU } \\
\text { Hai ou }\end{array}$ \\
\hline $\begin{array}{l}\text { j our nal or } \\
\text { publ i cat i on ti t e }\end{array}$ & The Quart er l y J our nal of Nat hemat i cs \\
\hline vol une & 69 \\
\hline number & 2 \\
\hline page r ange & $631-653$ \\
\hline year & 2018 12-14 \\
\hline URL & ht t p: //hdl . handl e. net /10258/00009670 \\
\hline
\end{tabular}




\title{
Envelopes of Legendre curves in the unit spherical bundle over the unit sphere
}

\author{
Yanlin $\mathrm{Li}^{\mathrm{a}, 1}$, Donghe Pei ${ }^{\mathrm{a}, 2}$, Masatomo Takahashi ${ }^{\mathrm{b}, 3}$, Haiou $\mathrm{Yu}^{\mathrm{a}, \mathrm{c}, *}$ \\ ${ }^{a}$ School of Mathematics and Statistics, Northeast Normal University, Changchun Jilin, 130024, China \\ ${ }^{b}$ Muroran Institute of Technology, Muroran, 050-8585, Japan \\ ${ }^{c}$ Department of Mathematical Education, College of Humanities and Sciences of Northeast Normal University, Changchun Jilin, \\ 130117, People's Republic of China
}

\begin{abstract}
In this paper, we introduce a one-parameter family of Legendre curves in the unit spherical bundle over the unit sphere and the curvature. We give the existence and uniqueness theorems for one-parameter families of spherical Legendre curves by using the curvatures. Then we define an envelope for the one-parameter family of Legendre curves in the unit spherical bundle. We also consider the parallel curves and evolutes of one-parameter families of Legendre curves in the unit spherical bundle and their envelopes. Moreover, we give relationships among one-parameter families of Legendre curves in the unit spherical bundle over the unit sphere and one-parameter families of Legendre curves in the unit tangent bundle over the Euclidean plane.
\end{abstract}

Keywords: Envelope, Spherical Legendre curve, Legendre curve, Singularity

2000 MSC: 58K05, 53A40, 57R45

\section{Introduction}

An envelope of a family of curves in the Euclidean plane is a curve that is tangent to each member of the family at some point. If the curves are regular, then the tangent is well-defined (cf. $[3,4,5,6,9])$. On the other hand, for singular plane curves, the classical definitions of envelopes are vague. In [11], the third author clarified the definition of the envelope for a family of singular curves in the unit tangent bundle over the Euclidean plane. This idea can be generalized to an envelope of a family of singular spherical curves. In [10], the third author gave a definition of Legendre curves in the unit spherical bundle, and established a moving frame of spherical Legendre curves. However, to the best of the authors knowledge, no literature exists regarding the envelope of a family of singular curves in the unit sphere. In this paper, we use these approach and techniques to give the definition and investigate properties of envelopes for families of Legendre curves in the unit spherical bundle over the unit sphere. For basic results on the singularity theory see $[1,2,4,7,8]$.

In section 2, we consider one-parameter families of Legendre curves in the unit spherical bundle and the curvatures. We give the existence and uniqueness theorems for one-parameter families of Legendre curves by using the curvatures. In section 3, we define an envelope of a one-parameter family of Legendre curves in the unit spherical bundle. We obtained that the envelope is also a Legendre curve. The envelope of the dual is the dual of the envelope of the one-parameter family of Legendre curves. We also give the definitions of parallel curves and evolutes of one-parameter families of Legendre curves in the unit sphere bundle. We found that the parallel curves and evolutes are also one-parameter families of Legendre curves in the unit sphere bundle, the evolutes if exists. Then we consider the envelopes of the parallel curves and evolutes. The envelope of parallel curves of a one-parameter family of Legendre curves is equal to the parallel curve of the envelope of the one-parameter family of Legendre curves. Under a condition, the envelope of the evolute of a one-parameter family of Legendre curves is equal to the evolute of the envelope of the one-parameter family of Legendre curves. In section 4, we give relationships among one-parameter families of Legendre curves in the unit spherical bundle over the unit

\footnotetext{
${ }^{*}$ Corresponding author

Email addresses: liy1744@nenu.edu.cn (Yanlin Li), peidh340@nenu.edu.cn (Donghe Pei), masatomo@mmm.muroran-it.ac.jp (Masatomo Takahashi), yuho930@nenu.edu.cn (Haiou Yu)

${ }^{1}$ Partially supported by CSC (Grant No. 201606620072).

${ }^{2}$ Partially supported by NSF of China (Grant No. 11671070).

${ }^{3}$ Partially supported by JSPS KAKENHI (Grant No. JP 17K05238).
} 
sphere and one-parameter families of Legendre curves in the unit tangent bundle over the Euclidean plane. In the last section 5, we give two examples and some pictures to illustrate our results.

All maps and manifolds considered here are differential of class $C^{\infty}$.

\section{Legendre curves in the unit spherical bundle over the unit sphere}

We first recall some definitions and theorems of Legendre curves in the unit spherical bundle over the unit sphere. For more detailed descriptions see [10].

Let $\mathbb{R}^{3}$ be the 3 -dimensional Euclidean space equipped with the inner product $\boldsymbol{a} \cdot \boldsymbol{b}=a_{1} b_{1}+a_{2} b_{2}+a_{3} b_{3}$, where $\boldsymbol{a}=\left(a_{1}, a_{2}, a_{3}\right)$ and $\boldsymbol{b}=\left(b_{1}, b_{2}, b_{3}\right) \in \mathbb{R}^{3}$. The vector product is given by

$$
\boldsymbol{a} \times \boldsymbol{b}=\left|\begin{array}{ccc}
\boldsymbol{e}_{1} & \boldsymbol{e}_{2} & \boldsymbol{e}_{3} \\
a_{1} & a_{2} & a_{3} \\
b_{1} & b_{2} & b_{3}
\end{array}\right|
$$

where $\boldsymbol{e}_{1}, \boldsymbol{e}_{2}, \boldsymbol{e}_{3}$ are the canonical basis on $\mathbb{R}^{3}$. Let $S^{2}=\left\{(x, y, z) \in \mathbb{R}^{3} \mid x^{2}+y^{2}+z^{2}=1\right\}$ be the unit sphere. We denote the set $\left\{(\boldsymbol{a}, \boldsymbol{b}) \in S^{2} \times S^{2} \mid \boldsymbol{a} \cdot \boldsymbol{b}=0\right\}$ by $\Delta$ (cf. [10]). Then $\Delta$ is a 3-dimensional smooth manifold.

We say that $(\gamma, \nu): I \rightarrow \Delta \subset S^{2} \times S^{2}$ is a Legendre curve (or, spherical Legendre curve) if $\dot{\gamma}(t) \cdot \nu(t)=0$ for all $t \in I$, that is, $(\gamma, \nu)$ is an integrable curve with respect to the canonical contact 1 -form on $\Delta$. We call $\gamma$ a frontal and $\nu$ a dual of $\gamma$. Moreover, if $(\gamma, \nu)$ is a Legendre immersion, we call $\gamma$ a front.

We define $\boldsymbol{\mu}(t)=\gamma(t) \times \nu(t)$. By definition, $\boldsymbol{\mu}(t) \in S^{2}, \gamma(t) \cdot \boldsymbol{\mu}(t)=0$ and $\nu(t) \cdot \boldsymbol{\mu}(t)=0$ for all $t \in I$. It follows that $\{\gamma(t), \nu(t), \boldsymbol{\mu}(t)\}$ is a moving frame along the frontal $\gamma(t)$.

Let $(\gamma, \nu): I \rightarrow \Delta$ be a Legendre curve. We have the Frenet type formula.

$$
\left(\begin{array}{c}
\dot{\gamma}(t) \\
\dot{\nu}(t) \\
\dot{\boldsymbol{\mu}}(t)
\end{array}\right)=\left(\begin{array}{ccc}
0 & 0 & m(t) \\
0 & 0 & n(t) \\
-m(t) & -n(t) & 0
\end{array}\right)\left(\begin{array}{c}
\gamma(t) \\
\nu(t) \\
\boldsymbol{\mu}(t)
\end{array}\right)
$$

where $m(t)=\dot{\gamma}(t) \cdot \boldsymbol{\mu}(t)$ and $n(t)=\dot{\nu}(t) \cdot \boldsymbol{\mu}(t)$.

We say that the pair of the functions $(m, n)$ is the curvature of the Legendre curve $(\gamma, \nu): I \rightarrow \Delta$.

Definition 2.1. Let $(\gamma, \nu),(\widetilde{\gamma}, \widetilde{\nu}): I \rightarrow \Delta$ be Legendre curves. We say that $(\gamma, \nu)$ and $(\widetilde{\gamma}, \widetilde{\nu})$ are congruent as Legendre curves if there exists a special orthogonal matrix $A \in S O(3)$ such that $\widetilde{\gamma}(t)=A(\gamma(t)), \widetilde{\nu}(t)=A(\nu(t))$ for all $t \in I$.

Then we have the following existence and uniqueness theorems in terms of the curvature of the Legendre curve [10].

Theorem 2.2 (The Existence Theorem of spherical Legendre curves). Let $(m, n): I \rightarrow \mathbb{R} \times \mathbb{R}$ be a smooth mapping. There exists a Legendre curve $(\gamma, \nu): I \rightarrow \Delta$, whose associated curvature is $(m, n)$.

Theorem 2.3 (The Uniqueness Theorem of spherical Legendre curves). Let $(\gamma, \nu)$ and $(\widetilde{\gamma}, \widetilde{\nu}): I \rightarrow \Delta$ be Legendre curves whose curvatures $(m, n)$ and $(\widetilde{m}, \widetilde{n})$, respectively. Then $(\gamma, \nu)$ and $(\widetilde{\gamma}, \widetilde{\nu})$ are congruent as Legendre curves if and only if $(m, n)$ and $(\widetilde{m}, \widetilde{n})$ coincides.

We consider one-parameter families of Legendre curves in the unit spherical bundle $\Delta \subset S^{2} \times S^{2}$. Let $I$ and $\Lambda$ be intervals of $\mathbb{R}$.

Definition 2.4. Let $(\gamma, \nu): I \times \Lambda \rightarrow \Delta$ be a smooth mapping. We say that $(\gamma, \nu)$ is a one-parameter family of spherical Legendre curves if $\gamma_{t}(t, \lambda) \cdot \nu(t, \lambda)=0$ for all $(t, \lambda) \in I \times \Lambda$.

By definition, $(\gamma(\cdot, \lambda), \nu(\cdot, \lambda)): I \rightarrow \Delta$ is a Legendre curve for each fixed parameter $\lambda \in \Lambda$.

We define $\boldsymbol{\mu}(t, \lambda)=\gamma(t, \lambda) \times \nu(t, \lambda)$. Then $\{\gamma(t, \lambda), \nu(t, \lambda), \boldsymbol{\mu}(t, \lambda)\}$ is a moving frame along the frontal $\gamma(t, \lambda)$ on $S^{2}$. We have the Frenet type formula.

$$
\left(\begin{array}{c}
\gamma_{t}(t, \lambda) \\
\nu_{t}(t, \lambda) \\
\boldsymbol{\mu}_{t}(t, \lambda)
\end{array}\right)=\left(\begin{array}{ccc}
0 & 0 & m(t, \lambda) \\
0 & 0 & n(t, \lambda) \\
-m(t, \lambda) & -n(t, \lambda) & 0
\end{array}\right)\left(\begin{array}{c}
\gamma(t, \lambda) \\
\nu(t, \lambda) \\
\boldsymbol{\mu}(t, \lambda)
\end{array}\right)
$$




$$
\left(\begin{array}{c}
\gamma_{\lambda}(t, \lambda) \\
\nu_{\lambda}(t, \lambda) \\
\boldsymbol{\mu}_{\lambda}(t, \lambda)
\end{array}\right)=\left(\begin{array}{ccc}
0 & L(t, \lambda) & M(t, \lambda) \\
-L(t, \lambda) & 0 & N(t, \lambda) \\
-M(t, \lambda) & -N(t, \lambda) & 0
\end{array}\right)\left(\begin{array}{c}
\gamma(t, \lambda) \\
\nu(t, \lambda) \\
\boldsymbol{\mu}(t, \lambda)
\end{array}\right)
$$

where

$$
\begin{aligned}
& m(t, \lambda)=\gamma_{t}(t, \lambda) \cdot \boldsymbol{\mu}(t, \lambda), \quad n(t, \lambda)=\nu_{t}(t, \lambda) \cdot \boldsymbol{\mu}(t, \lambda), \\
& L(t, \lambda)=\gamma_{\lambda}(t, \lambda) \cdot \nu(t, \lambda), \quad M(t, \lambda)=\gamma_{\lambda}(t, \lambda) \cdot \boldsymbol{\mu}(t, \lambda), \quad N(t, \lambda)=\nu_{\lambda}(t, \lambda) \cdot \boldsymbol{\mu}(t, \lambda) .
\end{aligned}
$$

We denote the matrices

$$
A(t, \lambda)=\left(\begin{array}{ccc}
0 & 0 & m(t, \lambda) \\
0 & 0 & n(t, \lambda) \\
-m(t, \lambda) & -n(t, \lambda) & 0
\end{array}\right), B(t, \lambda)=\left(\begin{array}{ccc}
0 & L(t, \lambda) & M(t, \lambda) \\
-L(t, \lambda) & 0 & N(t, \lambda) \\
-M(t, \lambda) & -N(t, \lambda) & 0
\end{array}\right)
$$

By $\gamma_{t \lambda}(t, \lambda)=\gamma_{\lambda t}(t, \lambda), \nu_{t \lambda}(t, \lambda)=\nu_{\lambda t}(t, \lambda)$ and $\boldsymbol{\mu}_{t \lambda}(t, \lambda)=\boldsymbol{\mu}_{\lambda t}(t, \lambda)$, we have the integrability condition $A_{\lambda}(t, \lambda)+A(t, \lambda) B(t, \lambda)=B_{t}(t, \lambda)+B(t, \lambda) A(t, \lambda)$, that is,

$$
\begin{aligned}
& L_{t}(t, \lambda)=M(t, \lambda) n(t, \lambda)-N(t, \lambda) m(t, \lambda), \\
& m_{\lambda}(t, \lambda)=M_{t}(t, \lambda)+L(t, \lambda) n(t, \lambda), \\
& n_{\lambda}(t, \lambda)=N_{t}(t, \lambda)-L(t, \lambda) m(t, \lambda)
\end{aligned}
$$

for all $(t, \lambda) \in \mathrm{I} \times \Lambda$. We call the tuple $(m, n, L, M, N)$ with the integrability condition $(2.1)$ the curvature of the one-parameter family of Legendre curves.

Remark 2.5. Let $(\gamma, \nu): I \times \Lambda \rightarrow \Delta$ be a one-parameter family of Legendre curves with the curvature $(m, n, L, M, N)$. Then it is easy to check that $(\gamma,-\nu),(-\gamma, \nu)$ and $(\nu, \gamma)$ are also one-parameter family of Legendre curves. The curvatures are $(-m, n,-L,-M, N),(m,-n,-L, M,-N)$ and $(-n,-m,-L,-N,-M)$ respectively.

Definition 2.6. Let $(\gamma, \nu)$ and $(\widetilde{\gamma}, \widetilde{\nu}): I \times \Lambda \rightarrow \Delta$ be one-parameter families of Legendre curves. We say that $(\gamma, \nu)$ and $(\widetilde{\gamma}, \widetilde{\nu})$ are congruent as one-parameter families of Legendre curves if there exists a special orthogonal matrix $A \in S O(3)$ such that $\widetilde{\gamma}(t, \lambda)=A(\gamma(t, \lambda))$ and $\widetilde{\nu}(t, \lambda)=A(\nu(t, \lambda))$ for all $(t, \lambda) \in I \times \Lambda$.

Then we have the following existence and uniqueness theorems for one-parameter families of Legendre curves.

Theorem 2.7 (The Existence Theorem for one-parameter families of spherical Legendre curves). Let $(m, n, L, M, N): I \times \Lambda \rightarrow \mathbb{R}^{5}$ be a smooth mapping with the integrability condition. There exists a one-parameter family of Legendre curves $(\gamma, \nu): I \times \Lambda \rightarrow \Delta$, whose associated curvature is $(m, n, L, M, N)$.

Proof. Choose any fixed value $t=t_{0}, \lambda=\lambda_{0}$ of the parameter. We consider an initial value problem,

$$
F_{t}(t, \lambda)=A(t, \lambda) F(t, \lambda), F_{\lambda}(t, \lambda)=B(t, \lambda) F(t, \lambda), F\left(t_{0}, \lambda_{0}\right)=I_{3},
$$

where $F(t, \lambda) \in M(3), A(t, \lambda), B(t, \lambda)$ as the above, $M(3)$ is the set of $3 \times 3$ matrices and $I_{3}$ is the identity matrix. Then we consider

$$
F_{t \lambda}=A_{\lambda} F+A F_{\lambda}=A_{\lambda} F+A B F=\left(A_{\lambda}+A B\right) F, F_{\lambda t}=B_{t} F+B F_{t}=B_{t} F+B A F=(B t+B A) F .
$$

By the integrability condition $A_{\lambda}+A B=B_{t}+B A$, we have $F_{t \lambda}=F_{\lambda t}$. Since $I \times \Lambda$ is simply connected, there exists a solution $F(t, \lambda)$. Therefore, there exists a one-parameter family of Legendre curves $(\gamma, \nu): I \times \Lambda \rightarrow \Delta$ whose associated curvature is $(m, n, L, M, N)$.

Lemma 2.8. Let $(\gamma, \nu)$ and $(\widetilde{\gamma}, \widetilde{\nu}): I \times \Lambda \rightarrow \Delta$ be one-parameter families of Legendre curves having equal curvature, that is, $(m(t, \lambda), n(t, \lambda), L(t, \lambda), M(t, \lambda), N(t, \lambda))=(\widetilde{m}(t, \lambda), \widetilde{n}(t, \lambda), \widetilde{L}(t, \lambda), \widetilde{M}(t, \lambda), \widetilde{N}(t, \lambda))$ for all $(t, \lambda) \in I \times \Lambda$. If there exist two parameters $t=t_{0}, \lambda=\lambda_{0}$ for which $\left(\gamma\left(t_{0}, \lambda_{0}\right), \nu\left(t_{0}, \lambda_{0}\right)\right)=\left(\widetilde{\gamma}\left(t_{0}, \lambda_{0}\right), \widetilde{\nu}\left(t_{0}, \lambda_{0}\right)\right)$, then $(\gamma, \nu)$ and $(\widetilde{\gamma}, \widetilde{\nu})$ coincides. 
Proof. Define a smooth function $f: I \times \Lambda \rightarrow \mathbb{R}$ by

$$
f(t, \lambda)=\gamma(t, \lambda) \cdot \widetilde{\gamma}(t, \lambda)+\nu(t, \lambda) \cdot \widetilde{\nu}(t, \lambda)+\boldsymbol{\mu}(t, \lambda) \cdot \widetilde{\boldsymbol{\mu}}(t, \lambda) .
$$

Since

$$
(m(t, \lambda), n(t, \lambda), L(t, \lambda), M(t, \lambda), N(t, \lambda))=(\widetilde{m}(t, \lambda), \widetilde{n}(t, \lambda), \widetilde{L}(t, \lambda), \widetilde{M}(t, \lambda), \widetilde{N}(t, \lambda))
$$

we have

$$
\begin{aligned}
f_{t}(t, \lambda)= & \left(\gamma_{t} \cdot \widetilde{\gamma}+\gamma \cdot \widetilde{\gamma}_{t}+\nu_{t} \cdot \widetilde{\nu}+\nu \cdot \widetilde{\nu}_{t}+\boldsymbol{\mu}_{t} \cdot \widetilde{\boldsymbol{\mu}}+\boldsymbol{\mu} \cdot \widetilde{\boldsymbol{\mu}}_{t}\right)(t, \lambda) \\
= & ((m \boldsymbol{\mu}) \cdot \widetilde{\gamma}+\gamma \cdot(\widetilde{m} \widetilde{\boldsymbol{\mu}})+(n \boldsymbol{\mu}) \cdot \widetilde{\nu}+\nu \cdot(\widetilde{n} \widetilde{\boldsymbol{\mu}})+(-m \gamma-n \nu) \cdot \widetilde{\boldsymbol{\mu}}+\boldsymbol{\mu} \cdot(-\widetilde{m} \widetilde{\gamma}-\widetilde{n} \widetilde{\nu}))(t, \lambda) \\
= & ((m-\widetilde{m}) \boldsymbol{\mu} \cdot \widetilde{\gamma}+(\widetilde{m}-m) \gamma \cdot \widetilde{\boldsymbol{\mu}}+(n-\widetilde{n}) \boldsymbol{\mu} \cdot \widetilde{\nu}+(\widetilde{n}-n) \nu \cdot \widetilde{\boldsymbol{\mu}})(t, \lambda) \\
= & 0 \\
f_{\lambda}(t, \lambda)= & \left(\gamma_{\lambda} \cdot \widetilde{\gamma}+\gamma \cdot \widetilde{\gamma}_{\lambda}+\nu_{\lambda} \cdot \widetilde{\nu}+\nu \cdot \widetilde{\nu}+\boldsymbol{\mu}_{\lambda} \cdot \widetilde{\boldsymbol{\mu}}+\boldsymbol{\mu} \cdot \widetilde{\boldsymbol{\mu}}_{\lambda}\right)(t, \lambda) \\
= & ((L \nu+M \boldsymbol{\mu}) \cdot \widetilde{\gamma}+\gamma \cdot(\widetilde{L} \widetilde{\nu}+\widetilde{M} \widetilde{\boldsymbol{\mu}})+(-L \gamma+N \boldsymbol{\mu}) \cdot \widetilde{\nu}+\nu \cdot(-\widetilde{L} \widetilde{\gamma}+\widetilde{N} \widetilde{\boldsymbol{\mu}}) \\
& +(-M \gamma-N \nu) \cdot \widetilde{\boldsymbol{\mu}}+\boldsymbol{\mu} \cdot(-\widetilde{M} \widetilde{\gamma}-\widetilde{N} \widetilde{\nu}))(t, \lambda) \\
= & ((L-\widetilde{L}) \nu \cdot \widetilde{\gamma}+(\widetilde{L}-L) \gamma \cdot \widetilde{\nu}+(M-\widetilde{M}) \boldsymbol{\mu} \cdot \widetilde{\gamma}+(\widetilde{M}-M) \gamma \cdot \widetilde{\boldsymbol{\mu}}+(N-\widetilde{N}) \boldsymbol{\mu} \cdot \widetilde{\nu}+(\widetilde{N}-N) \nu \cdot \widetilde{\boldsymbol{\mu}})(t, \lambda) \\
= & 0
\end{aligned}
$$

for all $(t, \lambda) \in I \times \Lambda$. It follows that $f$ is constant. By $\gamma\left(t_{0}, \lambda_{0}\right)=\widetilde{\gamma}\left(t_{0}, \lambda_{0}\right)$ and $\nu\left(t_{0}, \lambda_{0}\right)=\widetilde{\nu}\left(t_{0}, \lambda_{0}\right)$, we have $f\left(t_{0}, \lambda_{0}\right)=3$ and the function $f$ is constant with value 3 . By the Cauchy-Schwarz inequality, we have

$$
\begin{aligned}
& \gamma(t, \lambda) \cdot \widetilde{\gamma}(t, \lambda) \leqslant|\gamma(t, \lambda)||\widetilde{\gamma}(t, \lambda)|=1, \\
& \nu(t, \lambda) \cdot \widetilde{\nu}(t, \lambda) \leqslant|\nu(t, \lambda)||\widetilde{\nu}(t, \lambda)|=1, \\
& \boldsymbol{\mu}(t, \lambda) \cdot \widetilde{\boldsymbol{\mu}}(t, \lambda) \leqslant|\boldsymbol{\mu}(t, \lambda)||\widetilde{\boldsymbol{\mu}}(t, \lambda)|=1
\end{aligned}
$$

If one of these inequalities is strict, the value of $f(t, \lambda)$ would be less than 3 . It follows that these inequalities are equalities, and we have $\gamma(t, \lambda) \cdot \widetilde{\gamma}(t, \lambda)=1, \nu(t, \lambda) \cdot \widetilde{\nu}(t, \lambda)=1, \boldsymbol{\mu}(t, \lambda) \cdot \widetilde{\boldsymbol{\mu}}(t, \lambda)=1$ for all $(t, \lambda) \in I \times \Lambda$.

Then we have $|\gamma(t, \lambda)-\widetilde{\gamma}(t, \lambda)|^{2}=|\nu(t, \lambda)-\widetilde{\nu}(t, \lambda)|^{2}=|\boldsymbol{\mu}(t, \lambda)-\tilde{\boldsymbol{\mu}}(t, \lambda)|^{2}=0$. It follows that

$$
\gamma(t, \lambda)=\widetilde{\gamma}(t, \lambda), \nu(t, \lambda)=\widetilde{\nu}(t, \lambda), \boldsymbol{\mu}(t, \lambda)=\widetilde{\boldsymbol{\mu}}(t, \lambda) \text { for all }(t, \lambda) \in I \times \Lambda \text {. }
$$

Theorem 2.9 (The Uniqueness Theorem for one-parameter families of Legendre curves). Let $(\gamma, \nu)$ and $(\widetilde{\gamma}, \widetilde{\nu}): I \times \Lambda \rightarrow \Delta$ be one-parameter families of Legendre curves with the curvatures $(m, n, L, M, N)$ and $(\widetilde{m}, \widetilde{n}, \widetilde{L}, \widetilde{M}, \widetilde{N})$ respectively. Then $(\gamma, \nu)$ and $(\widetilde{\gamma}, \widetilde{\nu})$ are congruent as one-parameter family of Legendre curves if and only if $(m, n, L, M, N)$ and $(\widetilde{m}, \widetilde{n}, \widetilde{L}, \widetilde{M}, \widetilde{N})$ coincide.

Proof. Suppose that $(\gamma, \nu)$ and $(\widetilde{\gamma}, \widetilde{\nu})$ are congruent as one-parameter families of Legendre curves. By a direct calculation, we have

$$
\begin{aligned}
\widetilde{\gamma}_{t}(t, \lambda) & =\frac{\partial}{\partial t}(A(\gamma(t, \lambda)))=A\left(\gamma_{t}(t, \lambda)\right)=m(t, \lambda) A(\boldsymbol{\mu}(t, \lambda))=m(t, \lambda) \widetilde{\boldsymbol{\mu}}(t, \lambda), \\
\widetilde{\nu}_{t}(t, \lambda) & =\frac{\partial}{\partial t}(A(\nu(t, \lambda)))=A\left(\nu_{t}(t, \lambda)\right)=n(t, \lambda) A(\boldsymbol{\mu}(t, \lambda))=n(t, \lambda) \widetilde{\boldsymbol{\mu}}(t, \lambda), \\
\widetilde{\gamma}_{\lambda}(t, \lambda) & =\frac{\partial}{\partial \lambda}(A(\gamma(t, \lambda)))=A\left(\gamma_{\lambda}(t, \lambda)\right)=L(t, \lambda) A(\nu(t, \lambda))+M(t, \lambda) A(\boldsymbol{\mu}(t, \lambda)) \\
& =L(t, \lambda) \widetilde{\nu}(t, \lambda)+M(t, \lambda) \widetilde{\boldsymbol{\mu}}(t, \lambda), \\
\widetilde{\nu}_{\lambda}(t, \lambda) & =\frac{\partial}{\partial \lambda}(A(\nu(t, \lambda)))=A\left(\nu_{\lambda}(t, \lambda)\right)=-L(t, \lambda) A(\gamma(t, \lambda))+N(t, \lambda) A(\boldsymbol{\mu}(t, \lambda)) \\
& =-L(t, \lambda) \widetilde{\gamma}(t, \lambda)+N(t, \lambda) \widetilde{\boldsymbol{\mu}}(t, \lambda) .
\end{aligned}
$$

Therefore, the curvatures $(m, n, L, M, N)$ and $(\widetilde{m}, \widetilde{n}, \widetilde{L}, \widetilde{M}, \widetilde{N})$ coincide.

Conversely, suppose that $(m, n, L, M, N)$ and $(\widetilde{m}, \widetilde{n}, \widetilde{L}, \widetilde{M}, \widetilde{N})$ coincide. Let $\left(t_{0}, \lambda_{0}\right) \in I \times \Lambda$ be fixed. By using a congruence as one-parameter family of Legendre curves, we may assume $\gamma\left(t_{0}, \lambda_{0}\right)=\widetilde{\gamma}\left(t_{0}, \lambda_{0}\right)$ and $\nu\left(t_{0}, \lambda_{0}\right)=\widetilde{\nu}\left(t_{0}, \lambda_{0}\right)$. By Lemma 2.8, we have $\gamma(t, \lambda)=\widetilde{\gamma}(t, \lambda)$ and $\nu(t, \lambda)=\widetilde{\nu}(t, \lambda)$ for all $(t, \lambda) \in I \times \Lambda$. 


\section{Envelopes of one-parameter families of Legendre curves in the unit spherical bundle}

Let $(\gamma, \nu): I \times \Lambda \rightarrow \Delta$ be a one-parameter family of Legendre curves with the curvature $(m, n, L, M, N)$ and let $e: U \rightarrow I \times \Lambda, e(u)=(t(u), \lambda(u))$ be a smooth curve, where $U$ is an interval of $\mathbb{R}$. We denote $E_{\gamma}=\gamma \circ e: U \rightarrow S^{2}, E_{\gamma}(u)=\gamma \circ e(u)$ and $E_{\nu}=\nu \circ e: U \rightarrow S^{2}, E_{\nu}(u)=\nu \circ e(u)$.

Definition 3.1. We call $E_{\gamma}$ an envelope (and e a pre-envelope) for the one-parameter family of Legendre curves $(\gamma, \nu)$, when the following conditions are satisfied.

(i) The function $\lambda$ is non-constant on any non-trivial subinterval of $U$. (The Variability Condition.)

(ii) For all $u$, the curve $E_{\gamma}$ is tangent at $u$ to the curve $\gamma(t, \lambda)$ at the parameter $(t(u), \lambda(u))$, meaning that the tangent vectors $E_{\gamma}^{\prime}(u)=(d E / d u)(u)$ and $\boldsymbol{\mu}(t(u), \lambda(u))$ are linearly dependent. (The Tangency Condition.)

Note that the tangency condition is equivalent to the condition $E_{\gamma}^{\prime}(u) \cdot \nu(t(u), \lambda(u))=E_{\gamma}^{\prime}(u) \cdot E_{\nu}(u)=0$ for all $u \in U$. Therefore we have the following Proposition.

Proposition 3.2. Let $(\gamma, \nu): I \times \Lambda \rightarrow \Delta$ be a one-parameter family of Legendre curves with the curvature $(m, n, L, M, N)$. Suppose that $e: U \rightarrow I \times \Lambda, e(u)=(t(u), \lambda(u))$ is a pre-envelope and $E_{\gamma}=\gamma \circ e: U \rightarrow S^{2}$ is an envelope of $(\gamma, \nu)$. Then $E_{\gamma}$ is a frontal. More precisely, $\left(E_{\gamma}, E_{\nu}\right): U \rightarrow \Delta$ is a Legendre curve with the curvature

$$
m_{E_{\gamma}}(u)=t^{\prime}(u) m(e(u))+\lambda^{\prime}(u) M(e(u)), \quad n_{E_{\gamma}}(u)=t^{\prime}(u) n(e(u))+\lambda^{\prime}(u) N(e(u)) .
$$

Proof. By definition, $E_{\gamma}(u) \cdot E_{\nu}(u)=\gamma(e(u)) \cdot \nu(e(u))=0$ for all $u \in U$. Since $E_{\gamma}$ is an envelope, $E_{\gamma}^{\prime}(u) \cdot E_{\nu}(u)=0$ for all $u \in U$. It follows that $\left(E_{\gamma}, E_{\nu}\right): U \rightarrow \Delta$ is a Legendre curve. Then

$$
\begin{aligned}
& m_{E_{\gamma}(u)}=E_{\gamma}^{\prime}(u) \cdot \boldsymbol{\mu}(e(u))=\left(t^{\prime}(u) \gamma_{t}(e(u))+\lambda^{\prime}(u) \gamma_{\lambda}(e(u))\right) \cdot \boldsymbol{\mu}(e(u))=t^{\prime}(u) m(e(u))+\lambda^{\prime}(u) M(e(u)), \\
& n_{E_{\gamma}(u)}=E_{\nu}^{\prime}(u) \cdot \boldsymbol{\mu}(e(u))=\left(t^{\prime}(u) \nu_{t}(e(u))+\lambda^{\prime}(u) \nu_{\lambda}(e(u))\right) \cdot \boldsymbol{\mu}(e(u))=t^{\prime}(u) n(e(u))+\lambda^{\prime}(u) N(e(u))
\end{aligned}
$$

By Proposition 3.2, the envelope of the dual is the dual of the envelope of the one-parameter family of Legendre curves. We have the envelope theorem as follows:

Theorem 3.3. Let $(\gamma, \nu): I \times \Lambda \rightarrow \Delta$ be a one-parameter family of Legendre curves and let $e: U \rightarrow I \times \Lambda$ be a smooth curve satisfying the variability condition. Then $e$ is a pre-envelope of $(\gamma, \nu)$ (and $E_{\gamma}$ is an envelope) if and only if $\gamma_{\lambda}(e(u)) \cdot \nu(e(u))=0$ for all $u \in U$.

Proof. Suppose that $e$ is a pre-envelope of $(\gamma, \nu)$. By the tangency condition, there exists a function $c(u) \in \mathbb{R}$ such that $E_{\gamma}^{\prime}(u)=c(u) \boldsymbol{\mu}(e(u))$. By differentiating $E_{\gamma}(u)=\gamma \circ e(u)$, we have

$$
E_{\gamma}^{\prime}(u)=t^{\prime}(u) \gamma_{t}(e(u))+\lambda^{\prime}(u) \gamma_{\lambda}(e(u)) .
$$

It follows from $\gamma_{t}(t, \lambda)=m(t, \lambda) \boldsymbol{\mu}(t, \lambda)$ that $\left(t^{\prime}(u) m(e(u))-c(u)\right) \boldsymbol{\mu}(e(u))+\lambda^{\prime}(u) \gamma_{\lambda}(e(u))=0$. Then we have $\lambda^{\prime}(u) \gamma_{\lambda}(e(u)) \cdot \nu(e(u))=0$. By the variability condition, we have $\gamma_{\lambda}(e(u)) \cdot \nu(e(u))=0$ for all $u \in U$.

Conversely, suppose that $\gamma_{\lambda}(e(u)) \cdot \nu(e(u))=0$ for all $u \in U$. Since

$$
E_{\gamma}^{\prime}(u) \cdot \nu(e(u))=\left(t^{\prime}(u) \gamma_{t}(e(u))+\lambda^{\prime}(u) \gamma_{\lambda}(e(u))\right) \cdot \nu(e(u))=0
$$

$e$ is a pre-envelope of $(\gamma, \nu)$.

By using the curvature of the one-parameter family of Legendre curves, we have the corollary of Theorem 3.3 .

Corollary 3.4. Let $(\gamma, \nu): I \times \Lambda \rightarrow \Delta$ be a one-parameter family of Legendre curves with the curvature $(m, n, L, M, N)$ and let $e: U \rightarrow I \times \Lambda$ be a smooth curve satisfying the variability condition. Then $e: U \rightarrow I \times \Lambda$ is a pre-envelope of $(\gamma, \nu)$ (and $E_{\gamma}$ is an envelope) if and only if $L(e(u))=0$ for all $u \in U$.

Proposition 3.5. Let $(\gamma, \nu): I \times \Lambda \rightarrow \Delta$ be a one-parameter family of Legendre curves. Suppose that $e: U \rightarrow I \times \Lambda$ is a pre-envelope and $E_{\gamma}$ is an envelope of $(\gamma, \nu)$. Then $e: U \rightarrow I \times \Lambda$ is also a pre-envelope of $(-\gamma, \nu),(\gamma,-\nu)$ and $(\nu, \gamma)$. Moreover, $-E_{\gamma}$ is an envelope of $(-\gamma, \nu), E_{\gamma}$ is an envelope of $(\gamma,-\nu)$ and $E_{\nu}$ is an envelope of $(\nu, \gamma)$. 
Proof. Since $e: U \rightarrow I \times \Lambda$ is a pre-envelope, we have $\gamma_{\lambda}(e(u)) \cdot \nu(e(u))=0$ for all $u \in U$. It follows that $-\gamma_{\lambda}(e(u)) \cdot \nu(e(u))=0, \gamma_{\lambda}(e(u)) \cdot(-\nu(e(u)))=0$ and $\nu_{\lambda}(e(u)) \cdot \gamma(e(u))=0$ for all $u \in U$. Thus $e: U \rightarrow I \times \Lambda$ is also a pre-envelope of $(-\gamma, \nu),(\gamma,-\nu)$ and $(\nu, \gamma)$. It follows that $-E_{\gamma}=-\gamma \circ e, E_{\gamma}=\gamma \circ e$ and $E_{\nu}=\nu \circ e$ are envelopes of $(-\gamma, \nu),(\gamma,-\nu)$ and $(\nu, \gamma)$ respectively.

Definition 3.6. We say that a map $\Phi: \widetilde{I} \times \widetilde{\Lambda} \rightarrow I \times \Lambda$ is a one-parameter family of parameter change if $\Phi$ is a diffeomorphism and given by the form $\Phi(s, k)=(\phi(s, k), \varphi(k))$.

Proposition 3.7. Let $(\gamma, \nu): I \times \Lambda \rightarrow \Delta$ be a one-parameter family of Legendre curves with the curvature $(m, n, L, M, N)$. Suppose that $\Phi: \widetilde{I} \times \widetilde{\Lambda} \rightarrow I \times \Lambda$ is a one-parameter family of parameter change. Then $(\widetilde{\gamma}, \widetilde{\nu})=(\gamma \circ \Phi, \nu \circ \Phi): \widetilde{I} \times \widetilde{\Lambda} \rightarrow \Delta$ is also a one-parameter family of Legendre curves with the curvature

$$
\begin{aligned}
\widetilde{m}(s, k) & =m(\Phi(s, k)) \phi_{s}(s, k), \\
\widetilde{n}(s, k) & =n(\Phi(s, k)) \phi_{s}(s, k), \\
\widetilde{L}(s, k) & =L(\Phi(s, k)) \varphi^{\prime}(k), \\
\widetilde{M}(s, k) & =m(\Phi(s, k)) \phi_{k}(s, k)+M(\Phi(s, k)) \varphi^{\prime}(k), \\
\widetilde{N}(s, k) & =n(\Phi(s, k)) \phi_{k}(s, k)+N(\Phi(s, k)) \varphi^{\prime}(k) .
\end{aligned}
$$

If $e: U \rightarrow I \times \Lambda$ is a pre-envelope, $E_{\gamma}$ is an envelope, then $\Phi^{-1} \circ e: U \rightarrow \widetilde{I} \times \widetilde{\Lambda}$ is a pre-envelope and $E_{\gamma}$ is also an envelope of $(\widetilde{\gamma}, \widetilde{\nu})$.

Proof. Since $\widetilde{\gamma}_{s}(s, k)=\gamma_{t}(\Phi(s, k)) \phi_{s}(s, k)$ and $\gamma_{t}(t, \lambda) \cdot \nu(t, \lambda)=0$ for all $(t, \lambda) \in I \times \Lambda$, we have

$$
\widetilde{\gamma}_{s}(s, k) \cdot \widetilde{\nu}(s, k)=0 \text { for all }(s, k) \in \widetilde{I} \times \widetilde{\Lambda} .
$$

Therefore, $(\widetilde{\gamma}, \widetilde{\nu})$ is a one-parameter family of Legendre curves. Then we have

$$
\begin{aligned}
\widetilde{m}(s, k) & =\widetilde{\gamma}_{s}(s, k) \cdot \widetilde{\boldsymbol{\mu}}(s, k)=\gamma_{t}(\Phi(s, k)) \phi_{s}(s, k) \cdot \boldsymbol{\mu}(\Phi(s, k))=m(\Phi(s, k)) \phi_{s}(s, k), \\
\widetilde{n}(s, k) & =\widetilde{\nu}_{s}(s, k) \cdot \widetilde{\boldsymbol{\mu}}(s, k)=\nu_{t}(\Phi(s, k)) \phi_{s}(s, k) \cdot \boldsymbol{\mu}(\Phi(s, k))=n(\Phi(s, k)) \phi_{s}(s, k), \\
\widetilde{L}(s, k) & =\widetilde{\gamma}_{k}(s, k) \cdot \widetilde{\nu}(s, k)=\left(\gamma_{t}(\Phi(s, k)) \phi_{k}(s, k)+\gamma_{\lambda}(\Phi(s, k)) \varphi^{\prime}(k)\right) \cdot \nu(\Phi(s, k)) \\
& =L(\Phi(s, k)) \varphi^{\prime}(k), \\
\widetilde{M}(s, k) & =\widetilde{\gamma}_{k}(s, k) \cdot \widetilde{\boldsymbol{\mu}}(s, k)=\left(\gamma_{t}(\Phi(s, k)) \phi_{k}(s, k)+\gamma_{\lambda}(\Phi(s, k)) \varphi^{\prime}(k)\right) \cdot \boldsymbol{\mu}(\Phi(s, k)) \\
& =m(\Phi(s, k)) \phi_{k}(s, k)+M(\Phi(s, k)) \varphi^{\prime}(k), \\
\widetilde{N}(s, k) & =\widetilde{\nu}_{k}(s, k) \cdot \widetilde{\boldsymbol{\mu}}(s, k)=\left(\nu_{t}(\Phi(s, k)) \phi_{k}(s, k)+\nu_{\lambda}(\Phi(s, k)) \varphi^{\prime}(k)\right) \cdot \boldsymbol{\mu}(\Phi(s, k)) \\
& =n(\Phi(s, k)) \phi_{k}(s, k)+N(\Phi(s, k)) \varphi^{\prime}(k) .
\end{aligned}
$$

By the form of the diffeomorphism $\Phi(s, k)=(\phi(s, k), \varphi(k)), \Phi^{-1}: I \times \Lambda \rightarrow \widetilde{I} \times \widetilde{\Lambda}$ is given by the form $\Phi^{-1}(t, \lambda)=\left(\psi(t, \lambda), \varphi^{-1}(\lambda)\right)$. It follows that $\Phi^{-1} \circ e(u)=\left(\psi(t(u), \lambda(u)), \varphi^{-1}(\lambda(u))\right)$. Since

$$
(d / d u) \varphi^{-1}(\lambda(u))=\varphi_{\lambda}^{-1}(\lambda(u)) \lambda^{\prime}(u),
$$

the variability condition holds. Moreover, we have

$$
\widetilde{\gamma}_{k}(s, k) \cdot \widetilde{\nu}(s, k)=\left(\gamma_{t}(\Phi(s, k)) \phi_{k}(s, k)+\gamma_{\lambda}(\Phi(s, k)) \varphi^{\prime}(k)\right) \cdot \nu(\Phi(s, k))=\varphi^{\prime}(k) \gamma_{\lambda}(\Phi(s, k)) \cdot \nu(\Phi(s, k)) .
$$

It follows that

$$
\widetilde{\gamma}_{k}\left(\varphi^{-1} \circ e(u)\right) \cdot \widetilde{\nu}\left(\varphi^{-1} \circ e(u)\right)=\varphi^{\prime}\left(\varphi^{-1}(\lambda(u))\right) \gamma_{\lambda}(e(u)) \cdot \nu(e(u))=0 \text { for al } l u \in U
$$

By Theorem 3.3, $\Phi^{-1} \circ e$ is a pre-envelope of $(\widetilde{\gamma}, \widetilde{\nu})$. Therefore, $\widetilde{\gamma} \circ \Phi^{-1} \circ e=\gamma \circ \Phi \circ \Phi^{-1} \circ e=\gamma \circ e=E_{\gamma}$ is also an envelope of $(\widetilde{\gamma}, \widetilde{\nu})$.

Definition 3.8. Let $(\gamma, \nu): I \times \Lambda \rightarrow \Delta$ be a one-parameter family of Legendre curves, we define the parallel curves of the one-parameter family of Legendre curves by

$$
\gamma^{\theta}(t, \lambda)=\cos \theta \gamma(t, \lambda)-\sin \theta \nu(t, \lambda), \quad \nu^{\theta}(t, \lambda)=\sin \theta \gamma(t, \lambda)+\cos \theta \nu(t, \lambda) .
$$


Proposition 3.9. Let $(\gamma, \nu): I \times \Lambda \rightarrow \Delta$ be a one-parameter family of Legendre curves with the curvature $(m, n, L, M, N)$, then $\left(\gamma^{\theta}, \nu^{\theta}\right): I \times \Lambda \rightarrow \Delta$ is also a one-parameter family of Legendre curves with the curvature

$$
\begin{aligned}
& m^{\theta}(t, \lambda)=\cos \theta m(t, \lambda)-\sin \theta n(t, \lambda), n^{\theta}(t, \lambda)=\sin \theta m(t, \lambda)+\cos \theta n(t, \lambda), \\
& L^{\theta}(t, \lambda)=L(t, \lambda), M^{\theta}(t, \lambda)=\cos \theta M(t, \lambda)-\sin \theta N(t, \lambda), N^{\theta}(t, \lambda)=\sin \theta M(t, \lambda)+\cos \theta N(t, \lambda) .
\end{aligned}
$$

If $e: U \rightarrow I \times \Lambda$ is a pre-envelope of $(\gamma, \nu)$, then $e: U \rightarrow I \times \Lambda$ is also a pre-envelope of $\left(\gamma^{\theta}, \nu^{\theta}\right)$. Moreover, we have $\left(E_{\gamma}^{\theta}(u), E_{\nu}^{\theta}(u)\right)=\left(E_{\gamma^{\theta}}(u), E_{\nu^{\theta}}(u)\right)$ for all $u \in U$, where $\left(E_{\gamma}^{\theta}, E_{\nu}^{\theta}\right)$ is parallel curve of $\left(E_{\gamma}, E_{\nu}\right)$ and $\left(E_{\gamma^{\theta}}, E_{\gamma^{\theta}}\right)=\left(\gamma^{\theta} \circ e, \nu^{\theta} \circ e\right)$.

Proof. Since $(\gamma, \nu): I \times \Lambda \rightarrow \Delta$ is a one-parameter family of Legendre curves, $\gamma_{t}(t, \lambda) \cdot \nu(t, \lambda)=0$ for all $(t, \lambda) \in I \times \Lambda$. It follows that $\gamma_{t}^{\theta}(t, \lambda) \cdot \nu^{\theta}(t, \lambda)=0$ for all $(t, \lambda) \in I \times \Lambda$. Thus $\left(\gamma^{\theta}, \nu^{\theta}\right): I \times \Lambda \rightarrow \Delta$ is also a one-parameter family of Legendre curves. By definition,

$$
\boldsymbol{\mu}^{\theta}(t, \lambda)=\gamma^{\theta}(t, \lambda) \times \nu^{\theta}(t, \lambda)=(\cos \theta \gamma(t, \lambda)-\sin \theta \nu(t, \lambda)) \times(\sin \theta \gamma(t, \lambda)+\cos \theta \nu(t, \lambda))=\boldsymbol{\mu}(t, \lambda) .
$$

Therefore, we have

$$
\begin{aligned}
m^{\theta}(t, \lambda) & =\gamma_{t}^{\theta}(t, \lambda) \cdot \boldsymbol{\mu}^{\theta}(t, \lambda)=\cos \theta m(t, \lambda)-\sin \theta n(t, \lambda), \\
n^{\theta}(t, \lambda) & =\nu_{t}^{\theta}(t, \lambda) \cdot \boldsymbol{\mu}^{\theta}(t, \lambda)=\sin \theta m(t, \lambda)+\cos \theta n(t, \lambda), \\
L^{\theta}(t, \lambda) & =\gamma_{\lambda}^{\theta}(t, \lambda) \cdot \nu^{\theta}(t, \lambda)=L(t, \lambda), \\
M^{\theta}(t, \lambda) & =\gamma_{\lambda}^{\theta}(t, \lambda) \cdot \boldsymbol{\mu}^{\theta}(t, \lambda)=\cos \theta M(t, \lambda)-\sin \theta N(t, \lambda), \\
N^{\theta}(t, \lambda) & =\gamma_{\lambda}^{\theta}(t, \lambda) \cdot \boldsymbol{\mu}^{\theta}(t, \lambda)=\sin \theta M(t, \lambda)+\cos \theta N(t, \lambda) .
\end{aligned}
$$

Since $e: U \rightarrow I \times \Lambda$ is a pre-envelope of $(\gamma, \nu)$, we have $\gamma_{\lambda}(e(u)) \cdot \nu(e(u))=0$ for all $u \in U$. It follows that $\gamma_{\lambda}^{\theta}(e(u)) \cdot \nu^{\theta}(e(u))=0$ for all $u \in U$. Thus $e: U \rightarrow I \times \Lambda$ is a pre-envelope of $\left(\gamma^{\theta}, \nu^{\theta}\right)$ by Theorem 3.3. Moreover,

$$
\begin{aligned}
& E_{\gamma}^{\theta}(u)=\cos \theta E_{\gamma}(u)-\sin \theta E_{\nu}(u)=\cos \theta \gamma \circ e(u)-\sin \theta \nu \circ e(u), \\
& E_{\nu}^{\theta}(u)=\sin \theta E_{\gamma}(u)+\cos \theta E_{\nu}(u)=\sin \theta \gamma \circ e(u)+\cos \theta \nu \circ e(u), \\
& E_{\gamma^{\theta}}(u)=\gamma^{\theta} \circ e(u)=(\cos \theta \gamma-\sin \theta \nu) \circ e(u)=\cos \theta \gamma \circ e(u)-\sin \theta \nu \circ e(u), \\
& E_{\nu^{\theta}}(u)=\nu^{\theta} \circ e(u)=(\sin \theta \gamma+\cos \theta \nu) \circ e(u)=\sin \theta \gamma \circ e(u)+\cos \theta \nu \circ e(u) .
\end{aligned}
$$

Thus, we have $\left(E_{\gamma}^{\theta}(u), E_{\nu}^{\theta}(u)\right)=\left(E_{\gamma^{\theta}}(u), E_{\nu^{\theta}}(u)\right)$ for all $u \in U$.

In [10], the evolute of the spherical Legendre curve is defined. Now, we define the evolute of a one-parameter family of Legendre curves in the unit sphere bundle.

Definition 3.10. Let $(\gamma, \nu): I \times \Lambda \rightarrow \Delta$ be a one-parameter family of Legendre curves with the curvature $(m, n, L, M, N)$. Suppose that $(m(t, \lambda), n(t, \lambda)) \neq(0,0)$ for all $(t, \lambda) \in I \times \Lambda$. We define the evolute of the one-parameter family of Legendre curves $(\gamma, \nu)$ by

$$
\mathcal{E}(\gamma)(t, \lambda)= \pm \frac{n(t, \lambda)}{\sqrt{m^{2}(t, \lambda)+n^{2}(t, \lambda)}} \gamma(t, \lambda) \mp \frac{m(t, \lambda)}{\sqrt{m^{2}(t, \lambda)+n^{2}(t, \lambda)}} \nu(t, \lambda) .
$$

Proposition 3.11. Let $(\gamma, \nu): I \times \Lambda \rightarrow \Delta$ be a one-parameter family of Legendre curves with the curvature $(m, n, L, M, N)$. Suppose that $(m(t, \lambda), n(t, \lambda)) \neq(0,0)$, for all $(t, \lambda) \in I \times \Lambda$. Then the evolute $(\mathcal{E}(\gamma), \boldsymbol{\mu}): I \times \Lambda \rightarrow \Delta$ of $(\gamma, \nu)$ is also a one-parameter family of Legendre curves with the curvature $\left(m_{\mathcal{E}}, n_{\mathcal{E}}, L_{\mathcal{E}}, M_{\mathcal{E}}, N_{\mathcal{E}}\right)$, where

$$
\begin{aligned}
& m_{\mathcal{E}}(t, \lambda)=\frac{m_{t} n-m n_{t}}{m^{2}+n^{2}}(t, \lambda), n_{\mathcal{E}}(t, \lambda)= \pm \sqrt{m^{2}+n^{2}}(t, \lambda), \\
& L_{\mathcal{E}}(t, \lambda)= \pm \frac{n M-m N}{\sqrt{m^{2}+n^{2}}}(t, \lambda), M_{\mathcal{E}}(t, \lambda)=\frac{m_{\lambda} n-m n_{\lambda}-L\left(m^{2}+n^{2}\right)}{m^{2}+n^{2}}(t, \lambda), N_{\mathcal{E}}(t, \lambda)= \pm \frac{m M+n N}{\sqrt{m^{2}+n^{2}}}(t, \lambda) .
\end{aligned}
$$

If $e: U \rightarrow I \times \Lambda$ is a pre-envelope of $(\gamma, \nu)$ and $(n M-m N) \circ(e(u))=0$ for all $u \in U$, then $e: U \rightarrow I \times \Lambda$ is also a pre-envelope of $(\mathcal{E}(\gamma), \boldsymbol{\mu})$. Moreover, we have $E_{\mathcal{E}(\gamma)}(u)=\mathcal{E}_{E_{\gamma}}(u)$ for all $u \in U$, where $E_{\mathcal{E}(\gamma)}$ is the envelope of $\mathcal{E}(\gamma), \mathcal{E}_{E_{\gamma}}$ is the evolute of $E_{\gamma}$. 
Proof. Since $(\gamma, \nu): I \times \Lambda \rightarrow \Delta$ is a one-parameter family of Legendre curves and $\{\gamma(t, \lambda), \nu(t, \lambda), \boldsymbol{\mu}(t, \lambda)\}$ is a moving frame along the frontal $\gamma(t, \lambda)$, we have $\mathcal{E}(\gamma)(t, \lambda) \cdot \boldsymbol{\mu}(t, \lambda)=0, \mathcal{E}_{t}(\gamma)(t, \lambda) \cdot \boldsymbol{\mu}(t, \lambda)=0$ for all $(t, \lambda) \in I \times \Lambda$. Therefore $(\mathcal{E}(\gamma), \boldsymbol{\mu}): I \times \Lambda \rightarrow \Delta$ is a one-parameter family of Legendre curves. We denote $\left(\gamma_{\mathcal{E}}, \nu_{\mathcal{E}}\right)=(\mathcal{E}(\gamma), \boldsymbol{\mu})$. By definition

$$
\boldsymbol{\mu}_{\mathcal{E}}(t, \lambda)=\gamma_{\mathcal{E}}(t, \lambda) \times \nu_{\mathcal{E}}(t, \lambda)=\mp \frac{m(t, \lambda)}{\sqrt{m^{2}(t, \lambda)+n^{2}(t, \lambda)}} \gamma(t, \lambda) \mp \frac{n(t, \lambda)}{\sqrt{m^{2}(t, \lambda)+n^{2}(t, \lambda)}} \nu(t, \lambda) .
$$

Thus

$$
\begin{aligned}
& m_{\mathcal{E}}(t, \lambda)=\gamma_{\mathcal{E}_{t}}(t, \lambda) \cdot \boldsymbol{\mu}_{\mathcal{E}}(t, \lambda)=\frac{m_{t} n-m n_{t}}{m^{2}+n^{2}}(t, \lambda), \\
& n_{\mathcal{E}}(t, \lambda)=\nu_{\mathcal{E} t}(t, \lambda) \cdot \boldsymbol{\mu}_{\mathcal{E}}(t, \lambda)= \pm \sqrt{m^{2}+n^{2}}(t, \lambda), \\
& L_{\mathcal{E}}(t, \lambda)=\gamma_{\mathcal{E}_{\lambda}}(t, \lambda) \cdot \nu_{\mathcal{E}}(t, \lambda)= \pm \frac{n M-m N}{\sqrt{m^{2}+n^{2}}}(t, \lambda), \\
& M_{\mathcal{E}}(t, \lambda)=\gamma_{\mathcal{E} \lambda}(t, \lambda) \cdot \boldsymbol{\mu}_{\mathcal{E}}(t, \lambda)=\frac{m_{\lambda} n-m n_{\lambda}-L\left(m^{2}+n^{2}\right)}{m^{2}+n^{2}}(t, \lambda), \\
& N_{\mathcal{E}}(t, \lambda)=\nu_{\mathcal{E} \lambda}(t, \lambda) \cdot \boldsymbol{\mu}_{\mathcal{E}}(t, \lambda)= \pm \frac{m M+n N}{\sqrt{m^{2}+n^{2}}}(t, \lambda) .
\end{aligned}
$$

Since $(n M-m N) \circ(e(u))=0$ for all $u \in U$, we have

$$
\mathcal{E}(\gamma)_{\lambda}(e(u)) \cdot \boldsymbol{\mu}(e(u))= \pm \frac{n M-m N}{\sqrt{m^{2}+n^{2}}} \circ e(u)=0 .
$$

By Theorem 3.3, $e: U \rightarrow I \times \Lambda$ is a pre-envelope of $(\mathcal{E}(\gamma), \boldsymbol{\mu})$. The envelope of $\mathcal{E}(\gamma)$ is given by

$$
\begin{aligned}
E_{\mathcal{E}(\gamma)}(u) & =\mathcal{E}(\gamma) \circ e(u)=\left( \pm \frac{m}{\sqrt{m^{2}+n^{2}}} \gamma \mp \frac{n}{\sqrt{m^{2}+n^{2}}} \nu\right) \circ e(u) \\
& = \pm \frac{m}{\sqrt{m^{2}+n^{2}}}(e(u)) \gamma(e(u)) \mp \frac{n}{\sqrt{m^{2}+n^{2}}}(e(u)) \nu(e(u)) .
\end{aligned}
$$

On the other hand, by Proposition 3.2, the evolute of $E_{\gamma}$ is given by

$$
\begin{aligned}
\mathcal{E}_{E_{\gamma}}(u) & = \pm \frac{m_{E_{\gamma}}(u)}{\sqrt{m_{E_{\gamma}}^{2}(u)+n_{E_{\gamma}}^{2}(u)}} E_{\gamma}(u) \mp \frac{n_{E_{\gamma}}(u)}{\sqrt{m_{E_{\gamma}}^{2}(u)+n_{E_{\gamma}}^{2}(u)}} E_{\nu}(u) \\
& = \pm \frac{t^{\prime} m+\lambda^{\prime} M}{\sqrt{\left(t^{\prime} m+\lambda^{\prime} M\right)^{2}+\left(t^{\prime} n+\lambda^{\prime} N\right)^{2}}}(e(u)) \gamma(e(u)) \mp \frac{t^{\prime} n+\lambda^{\prime} N}{\sqrt{\left(t^{\prime} m+\lambda^{\prime} M\right)^{2}+\left(t^{\prime} n+\lambda^{\prime} N\right)^{2}}}(e(u)) \nu(e(u)) .
\end{aligned}
$$

Since $(n M-m N)(e(u))=0$ for all $u \in U$, we have

$$
\begin{aligned}
\left(t^{\prime} m+\lambda^{\prime} M\right)^{2}\left(m^{2}+n^{2}\right)(e(u)) & =m^{2}\left(\left(t^{\prime} m+\lambda^{\prime} M\right)^{2}+\left(t^{\prime} n+\lambda^{\prime} N\right)^{2}\right)(e(u)) \\
\left(t^{\prime} n+\lambda^{\prime} N\right)^{2}\left(m^{2}+n^{2}\right)(e(u)) & =n^{2}\left(\left(t^{\prime} m+\lambda^{\prime} M\right)^{2}+\left(t^{\prime} n+\lambda^{\prime} N\right)^{2}\right)(e(u))
\end{aligned}
$$

Then

$$
\begin{aligned}
& \frac{t^{\prime} m+\lambda^{\prime} M}{\sqrt{\left(t^{\prime} m+\lambda^{\prime} M\right)^{2}+\left(t^{\prime} n+\lambda^{\prime} N\right)^{2}}}(e(u))=\frac{m}{\sqrt{m^{2}+n^{2}}}(e(u)), \\
& \frac{t^{\prime} n+\lambda^{\prime} N}{\sqrt{\left(t^{\prime} m+\lambda^{\prime} M\right)^{2}+\left(t^{\prime} n+\lambda^{\prime} N\right)^{2}}}(e(u))=\frac{n}{\sqrt{m^{2}+n^{2}}}(e(u)) .
\end{aligned}
$$

Thus, we have $E_{\mathcal{E}(\gamma)}(u)=\mathcal{E}_{E_{\gamma}}(u)$ for all $u \in U$.

\section{Relationships among envelopes of Legendre curves in the spherical bundle over the unit sphere and the unit tangent bundle over the Euclidean plane}

We first recall the definition of the envelope of a one-parameter family of Legendre curves in the unit tangent bundle over the Euclidean plane. For more detailed descriptions see [11]. 
Let $(\gamma, \nu): I \times \Lambda \rightarrow \mathbb{R}^{2} \times S^{1}$ be a smooth mapping. We say that $(\gamma, \nu)$ is a one-parameter family of Legendre curves if $\gamma_{t}(t, \lambda) \cdot \nu(t, \lambda)=0$ for all $(t, \lambda) \in I \times \Lambda$.

We denote $\boldsymbol{J}(a)=\left(-a_{2}, a_{1}\right)$ the anticlockwise rotation by $\pi / 2$ of a vector $\boldsymbol{a}=\left(a_{1}, a_{2}\right)$. We define $\boldsymbol{\mu}(t, \lambda)=$ $\boldsymbol{J}(\nu(t, \lambda))$. Since $\{\nu(t, \lambda), \boldsymbol{\mu}(t, \lambda)\}$ is a moving frame along $\gamma(t, \lambda)$ on $\mathbb{R}^{2}$, we have the Frenet type formula.

$$
\begin{aligned}
\left(\begin{array}{c}
\nu_{t}(t, \lambda) \\
\boldsymbol{\mu}_{t}(t, \lambda)
\end{array}\right) & =\left(\begin{array}{cc}
0 & \ell(t, \lambda) \\
-\ell(t, \lambda) & 0
\end{array}\right)\left(\begin{array}{c}
\nu(t, \lambda) \\
\boldsymbol{\mu}(t, \lambda)
\end{array}\right), \\
\left(\begin{array}{c}
\nu_{\lambda}(t, \lambda) \\
\boldsymbol{\mu}_{\lambda}(t, \lambda)
\end{array}\right) & =\left(\begin{array}{cc}
0 & m(t, \lambda) \\
-m(t, \lambda) & 0
\end{array}\right)\left(\begin{array}{c}
\nu(t, \lambda) \\
\boldsymbol{\mu}(t, \lambda)
\end{array}\right), \\
\gamma_{t}(t, \lambda) & =\beta(t, \lambda) \boldsymbol{\mu}(t, \lambda),
\end{aligned}
$$

where $\ell(t, \lambda)=\nu_{t}(t, \lambda) \cdot \boldsymbol{\mu}(t, \lambda), m(t, \lambda)=\nu_{\lambda}(t, \lambda) \cdot \boldsymbol{\mu}(t, \lambda)$ and $\beta(t, \lambda)=\gamma_{t}(t, \lambda) \cdot \boldsymbol{\mu}(t, \lambda)$. By the integrability condition $\nu_{t \lambda}(t, \lambda)=\nu_{\lambda t}(t, \lambda), \ell$ and $m$ satisfy the condition $\ell_{\lambda}(t, \lambda)=m_{t}(t, \lambda)$ for all $(t, \lambda) \in I \times \Lambda$. We call the triple $(\ell, m, \beta)$ are the curvature of the one-parameter family of Legendre curves $(\gamma, \nu)$.

Let $(\gamma, \nu): I \times \Lambda \rightarrow \mathbb{R}^{2} \times S^{1}$ be a one-parameter family of Legendre curves with the curvature $(\ell, m, \beta)$ and let $e: U \rightarrow I \times \Lambda, e(u)=(t(u), \lambda(u))$ be a smooth curve, where $U$ is an interval of $\mathbb{R}$. We denote $E_{\gamma}=\gamma \circ e: U \rightarrow \mathbb{R}^{2}, E_{\gamma}(u)=\gamma \circ e(u)$ and $E_{\nu}=\nu \circ e: U \rightarrow \mathbb{R}^{2}, E_{\nu}(u)=\nu \circ e(u)$.

We call $E_{\gamma}$ an envelope (and $e$ a pre-envelope) for the one-parameter family of Legendre curves $(\gamma, \nu): I \times \Lambda \rightarrow \mathbb{R}^{2} \times S^{1}$, when the following conditions satisfy.

(i) The function $\lambda$ is non-constant on any non-trivial subinterval of $U$. (The Variability Condition.)

(ii) For all $u$, the curve $E_{\gamma}$ is tangent at $u$ to the curve $\gamma(t, \lambda)$ at the parameter $(t(u), \lambda(u))$, meaning that the tangent vectors $E_{\gamma}^{\prime}(u)=(d E / d u)(u)$ and $\boldsymbol{\mu}(t(u), \lambda(u))$ are linearly dependent. (The Tangency Condition.)

We consider relationships among envelopes of Legendre curves in the spherical bundle over the unit sphere and the unit tangent bundle over the Euclidean plane. We denote a hemisphere $S^{+}=\left\{(x, y, z) \in S^{2} \mid z>0\right\}$.

Now we consider the central projection $\phi: S^{+} \rightarrow \mathbb{R}^{2}$ by

$$
\phi(x, y, z)=\left(\frac{x}{z}, \frac{y}{z}\right)
$$

Proposition 4.1. Let $(\gamma, \nu): I \times \Lambda \rightarrow \Delta$ be a one-parameter family of Legendre curves with the curvature $(m, n, L, M, N)$ and $\gamma(I \times \Lambda) \subset S^{+}$. We denote $\gamma(t, \lambda)=(x(t, \lambda), y(t, \lambda), z(t, \lambda)), \nu(t, \lambda)=(a(t, \lambda), b(t, \lambda), c(t, \lambda))$. Suppose that $(a(t, \lambda), b(t, \lambda)) \neq(0,0)$ for all $(t, \lambda) \in I \times \Lambda$. Then $(\widetilde{\gamma}, \widetilde{\nu}): I \times \Lambda \rightarrow \mathbb{R}^{2} \times S^{1}$ is a one-parameter family of Legendre curves with the curvature $(\widetilde{\ell}, \widetilde{m}, \widetilde{\beta})$, where

$$
\begin{aligned}
& \widetilde{\gamma}(t, \lambda)=\phi \circ \gamma(t, \lambda)=\left(\frac{x(t, \lambda)}{z(t, \lambda)}, \frac{y(t, \lambda)}{z(t, \lambda)}\right), \widetilde{\nu}(t, \lambda)=\frac{1}{\sqrt{a^{2}(t, \lambda)+b^{2}(t, \lambda)}}(a(t, \lambda), b(t, \lambda)), \\
& \widetilde{\ell}(t, \lambda)=\frac{n z}{a^{2}+b^{2}}(t, \lambda), \widetilde{m}(t, \lambda)=\frac{N z}{a^{2}+b^{2}}(t, \lambda), \widetilde{\beta}(t, \lambda)=\frac{m z^{2}+(x b-y a) z_{t}}{z^{2} \sqrt{a^{2}+b^{2}}}(t, \lambda) .
\end{aligned}
$$

Proof. Since $(\gamma, \nu): I \times \Lambda \rightarrow \Delta$ is a one-parameter family of Legendre curves in the unit spherical bundle, we have $\gamma(t, \lambda) \cdot \nu(t, \lambda)=0, \gamma_{t}(t, \lambda) \cdot \nu(t, \lambda)=0$ for all $(t, \lambda) \in I \times \Lambda$. It follows that

$$
x(t, \lambda) a_{t}(t, \lambda)+y(t, \lambda) b_{t}(t, \lambda)+z(t, \lambda) c_{t}(t, \lambda)=0 .
$$

By definition, we have

$$
\begin{aligned}
\boldsymbol{\mu}(t, \lambda) & =\gamma(t, \lambda) \times \nu(t, \lambda) \\
& =(y(t, \lambda) c(t, \lambda)-z(t, \lambda) b(t, \lambda), z(t, \lambda) a(t, \lambda)-x(t, \lambda) c(t, \lambda), x(t, \lambda) b(t, \lambda)-y(t, \lambda) a(t, \lambda)) .
\end{aligned}
$$

By a direct calculation, we have

$$
\begin{gathered}
m(t, \lambda)=\gamma_{t}(t, \lambda) \cdot \boldsymbol{\mu}(t, \lambda)=\frac{-x_{t} b+y_{t} a}{z}(t, \lambda), \\
n(t, \lambda)=\nu_{t}(t, \lambda) \cdot \boldsymbol{\mu}(t, \lambda)=\frac{-a_{t} b+b_{t} a}{z}(t, \lambda), \\
N(t, \lambda)=\nu_{\lambda}(t, \lambda) \cdot \boldsymbol{\mu}(t, \lambda)=\frac{-a_{\lambda} b+b_{\lambda} a}{z}(t, \lambda) .
\end{gathered}
$$


By the assumption $(a(t, \lambda), b(t, \lambda)) \neq(0,0), \widetilde{\nu}: I \times \Lambda \rightarrow S^{1}$ is a smooth mapping. Moreover, we have

$$
\widetilde{\gamma}_{t}(t, \lambda)=\left(x_{t}(t, \lambda) z(t, \lambda)-x(t, \lambda) z_{t}(t, \lambda), y_{t}(t, \lambda) z(t, \lambda)-y(t, \lambda) z_{t}(t, \lambda)\right) / z^{2}(t, \lambda) \text { and } \widetilde{\gamma}_{t}(t, \lambda) \cdot \widetilde{\nu}(t, \lambda)=0 .
$$

Therefore $(\widetilde{\gamma}, \widetilde{\nu}): I \times \Lambda \rightarrow \mathbb{R}^{2} \times S^{1}$ is a one-parameter family of Legendre curves.

By definition, we have $\widetilde{\boldsymbol{\mu}}(t, \lambda)=\boldsymbol{J}(\widetilde{\nu}(t, \lambda))=(-b(t, \lambda), a(t, \lambda)) / \sqrt{a^{2}(t, \lambda)+b^{2}(t, \lambda)}$ and the curvature

$$
\begin{aligned}
\widetilde{\ell}(t, \lambda) & =\widetilde{\nu}_{t}(t, \lambda) \cdot \widetilde{\boldsymbol{\mu}}(t, \lambda)=\frac{-a_{t} b+b_{t} a}{a^{2}+b^{2}}(t, \lambda)=\frac{n z}{a^{2}+b^{2}}(t, \lambda), \\
\widetilde{m}(t, \lambda) & =\widetilde{\nu}_{\lambda}(t, \lambda) \cdot \widetilde{\boldsymbol{\mu}}(t, \lambda)=\frac{-a_{\lambda} b+b_{\lambda} a}{a^{2}+b^{2}}(t, \lambda)=\frac{N z}{a^{2}+b^{2}}(t, \lambda), \\
\widetilde{\beta}(t, \lambda) & =\widetilde{\gamma}_{t}(t, \lambda) \cdot \widetilde{\boldsymbol{\mu}}(t, \lambda)=\frac{\left(-x_{t} b+y_{t} a\right) z+(x b-y a) z_{t}}{z^{2} \sqrt{a^{2}+b^{2}}}(t, \lambda)=\frac{m z^{2}+(x b-y a) z_{t}}{z^{2} \sqrt{a^{2}+b^{2}}}(t, \lambda) .
\end{aligned}
$$

Proposition 4.2. Under the same assumptions in Proposition 4.1, suppose that $e: U \rightarrow I \times \Lambda$ is a pre-envelope of $(\gamma, \nu)$ and $E_{\gamma}: U \rightarrow S^{2}$ is an envelope. Then $e: U \rightarrow I \times \Lambda$ is also a pre-envelope of $(\widetilde{\gamma}, \widetilde{\nu}): I \times \Lambda \rightarrow \mathbb{R}^{2} \times S^{1}$. Moreover, we have $E_{\widetilde{\gamma}}(u)=\widetilde{E}_{\gamma}(u)$ for all $u \in U$, where $E_{\widetilde{\gamma}}=\widetilde{\gamma} \circ$ e and $\widetilde{E}_{\gamma}=\phi \circ E_{\gamma}$.

Proof. Since $(\gamma, \nu): I \times \Lambda \rightarrow \Delta$ is a one-parameter family of Legendre curves in the unit spherical bundle and $e: U \rightarrow I \times \Lambda$ is a pre-envelope of $(\gamma, \nu)$, we have $\gamma(t, \lambda) \cdot \nu(t, \lambda)=0$ for all $(t, \lambda) \in I \times \Lambda, \gamma_{\lambda}(e(u)) \cdot \nu(e(u))=0$ for all $u \in U$. It follows that

$$
\left(a(t, \lambda)\left(x_{\lambda}(t, \lambda) z(t, \lambda)-x(t, \lambda) z_{\lambda}(t, \lambda)\right)+b(t, \lambda)\left(y_{\lambda}(t, \lambda) z(t, \lambda)-y(t, \lambda) z_{\lambda}(t, \lambda)\right)\right) \circ e(u)=0 .
$$

Then we have $\widetilde{\gamma}_{\lambda}(e(u)) \cdot \widetilde{\nu}(e(u))=0$ for all $u \in U$. Therefore $e: U \rightarrow I \times \Lambda$ is a pre-envelope of $(\widetilde{\gamma}, \widetilde{\nu})(\mathrm{cf}$. [11]). Moreover, we have $E_{\widetilde{\gamma}}(u)=\widetilde{\gamma} \circ e(u)=\phi \circ \gamma \circ e(u)=\phi\left(E_{\gamma}(u)\right)=\widetilde{E}_{\gamma}(u)$ for all $u \in U$.

Conversely, we have the following results.

Proposition 4.3. Let $(\widetilde{\gamma}, \widetilde{\nu}): I \times \Lambda \rightarrow \mathbb{R}^{2} \times S^{1}$ be a one-parameter family of Legendre curves with the curvature $(\widetilde{\ell}, \widetilde{m}, \widetilde{\beta})$. We denote $\widetilde{\gamma}(t, \lambda)=(x(t, \lambda), y(t, \lambda)), \widetilde{\nu}(t, \lambda)=(a(t, \lambda), b(t, \lambda))$. Then $(\gamma, \nu): I \times \Lambda \rightarrow \Delta \subset S^{+} \times S^{2}$ is a one-parameter family of Legendre curves in the unit spherical bundle with the curvature $(m, n, L, M, N)$, where

$$
\begin{aligned}
\gamma(t, \lambda) & =\phi^{-1} \circ \widetilde{\gamma}(t, \lambda)=\frac{(x, y, 1)}{\sqrt{1+x^{2}+y^{2}}}(t, \lambda), \nu(t, \lambda)=\frac{(a, b,-x a-y b)}{\sqrt{1+(x a+y b)^{2}}}(t, \lambda) \\
m(t, \lambda) & =\frac{\widetilde{\beta}+\left(y_{t} x-x_{t} y\right)(x a+y b)}{\left(1+x^{2}+y^{2}\right) \sqrt{1+(x a+y b)^{2}}}(t, \lambda), n(t, \lambda)=\frac{\widetilde{\ell} \sqrt{1+x^{2}+y^{2}}}{1+(x a+y b)^{2}}(t, \lambda), \\
L(t, \lambda) & =\frac{x_{\lambda} a+y_{\lambda} b}{\sqrt{1+x^{2}+y^{2}} \sqrt{1+(x a+y b)^{2}}}(t, \lambda), M(t, \lambda)=\frac{\left(y_{\lambda} x-x_{\lambda} y\right)(x a+y b)+y_{\lambda} a-x_{\lambda} b}{\left(1+x^{2}+y^{2}\right) \sqrt{1+(x a+y b)^{2}}}(t, \lambda), \\
N(t, \lambda) & =\frac{\widetilde{m}\left(1+x^{2}+y^{2}\right)+\left(x_{\lambda} a+y_{\lambda} b\right)(x b-y a)}{\sqrt{1+x^{2}+y^{2}}\left(1+(x a+y b)^{2}\right)}(t, \lambda) .
\end{aligned}
$$

Proof. Since $(\widetilde{\gamma}, \widetilde{\nu}): I \times \Lambda \rightarrow \mathbb{R}^{2} \times S^{1}$ is a one-parameter family of Legendre curves, then we have

$$
\widetilde{\gamma}_{t}(t, \lambda) \cdot \widetilde{\nu}(t, \lambda)=\left(x_{t} a+y_{t} b\right)(t, \lambda)=0 \text { for all }(t, \lambda) \in I \times \Lambda \text {. }
$$

By the definition, $\widetilde{\boldsymbol{\mu}}(t, \lambda)=\boldsymbol{J}(\widetilde{\nu}(t, \lambda))=(-b(t, \lambda), a(t, \lambda))$. It follows that

$$
\tilde{\ell}(t, \lambda)=\left(-a_{t} b+a b_{t}\right)(t, \lambda), \widetilde{\beta}(t, \lambda)=\left(-x_{t} b+y_{t} a\right)(t, \lambda), \widetilde{m}(t, \lambda)=\left(-a_{\lambda} b+a b_{\lambda}\right)(t, \lambda) .
$$

By a direct calculation, we have

$$
\gamma_{t}(t, \lambda)=\frac{1}{1+x^{2}+y^{2}}\left(\left(1+y^{2}\right) x_{t}-x y y_{t},\left(1+x^{2}\right) y_{t}-x x_{t} y,-x x_{t}-y y_{t}\right)(t, \lambda)
$$


Then $\gamma(t, \lambda) \cdot \nu(t, \lambda)=0$ and $\gamma_{t}(t, \lambda) \cdot \nu(t, \lambda)=0$ for all $(t, \lambda) \in I \times \Lambda$. Therefore $(\gamma, \nu): I \times \Lambda \rightarrow \Delta$ is a one-parameter family of Legendre curves in the unit spherical bundle. By definition, $\boldsymbol{\mu}(t, \lambda)$ is given by

$$
\boldsymbol{\mu}(t, \lambda)=\gamma(t, \lambda) \times \nu(t, \lambda)=\frac{\left(-x y a-\left(1+y^{2}\right) b,\left(1+x^{2}\right) a+x y b, x b-y a\right)}{\sqrt{\left(1+x^{2}+y^{2}\right)\left(1+(x a+y b)^{2}\right)}}(t, \lambda) .
$$

By a direct calculation, we have the curvature $(m, n, L, M, N)$ of $(\gamma, \nu)$.

Proposition 4.4. Under the same assumptions in Proposition 4.3, suppose that $e: U \rightarrow I \times \Lambda$ is a pre-envelope of $(\widetilde{\gamma}, \widetilde{\nu})$ and $E_{\widetilde{\gamma}}: U \rightarrow \mathbb{R}^{2}$ is an envelope. Then $e: U \rightarrow I \times \Lambda$ is also a pre-envelope of $(\gamma, \nu): I \times \Lambda \rightarrow \Delta \subset S^{+} \times S^{2}$. Moreover, we have $\phi^{-1} \circ E_{\widetilde{\gamma}}(u)=E_{\gamma}(u)$ for all $u \in U$.

Proof. Since $e: U \rightarrow I \times \Lambda$ is a pre-envelope of $(\widetilde{\gamma}, \widetilde{\nu})$, we have $\widetilde{\gamma}_{\lambda}(e(u)) \cdot \widetilde{\nu}(e(u))=0$ for all $u \in U$ (cf. [11]). It follows that

$$
\left(x_{\lambda}(t, \lambda) \cdot a(t, \lambda)+y_{\lambda}(t, \lambda) \cdot b(t, \lambda)\right) \circ e(u)=0 .
$$

By a direct calculation, we have $\gamma_{\lambda}(e(u)) \cdot \nu(e(u))=0$ for all $u \in U$. By Theorem 3.3, $e: U \rightarrow I \times \Lambda$ is a pre-envelope of $(\gamma, \nu)$. Moreover, we have $\phi^{-1} \circ E_{\widetilde{\gamma}}(u)=\phi^{-1} \circ \widetilde{\gamma} \circ e(u)=\gamma \circ e(u)=E_{\gamma}(u)$ for all $u \in U$.

Also, we consider the canonical projection $\pi: S^{+} \rightarrow D^{2} \subset \mathbb{R}^{2}$ by $\pi(x, y, z)=(x, y)$, where

$$
D^{2}=\left\{(x, y) \in \mathbb{R}^{2} \mid x^{2}+y^{2}<1\right\} .
$$

Proposition 4.5. Let $(\gamma, \nu): I \times \Lambda \rightarrow \Delta$ be a one-parameter family of Legendre curves in the unit spherical bundle with the curvature $(m, n, L, M, N)$ and $\gamma(I \times \Lambda) \subset S^{+}$. We denote $\gamma(t, \lambda)=(x(t, \lambda), y(t, \lambda), z(t, \lambda))$ and $\nu(t, \lambda)=(a(t, \lambda), b(t, \lambda), c(t, \lambda))$. Then $(\widetilde{\gamma}, \widetilde{\nu}): I \times \Lambda \rightarrow D^{2} \times S^{1}$ is a one-parameter family of Legendre curves in the unit tangent bundle over the Euclidean plane with the curvature $(\widetilde{\ell}, \widetilde{m}, \widetilde{\beta})$, where

$$
\begin{aligned}
& \widetilde{\gamma}(t, \lambda)=\pi \circ \gamma(t, \lambda)=(x(t, \lambda), y(t, \lambda)), \widetilde{\nu}(t, \lambda)=\frac{(z a-x c, z b-y c)}{\sqrt{(z a-x c)^{2}+(z b-y c)^{2}}}(t, \lambda), \\
& \tilde{\ell}(t, \lambda)=\frac{n z+x y_{t}-x_{t} y}{(z a-x c)^{2}+(z b-y c)^{2}}(t, \lambda), \tilde{m}(t, \lambda)=\frac{N z+x y_{\lambda}-x_{\lambda} y}{(z a-x c)^{2}+(z b-y c)^{2}}(t, \lambda), \\
& \widetilde{\beta}(t, \lambda)=\frac{m-(x b-y a) z_{t}}{\sqrt{(z a-x c)^{2}+(z b-y c)^{2}}}(t, \lambda) .
\end{aligned}
$$

Proof. If $z(t, \lambda) a(t, \lambda)-x(t, \lambda) c(t, \lambda)=0$ and $z(t, \lambda) b(t, \lambda)-y(t, \lambda) c(t, \lambda)=0$, then

$$
a(t, \lambda)=x(t, \lambda) c(t, \lambda) / z(t, \lambda) \text { and } b(t, \lambda)=y(t, \lambda) c(t, \lambda) / z(t, \lambda) .
$$

Since $\nu(t, \lambda) \in S^{2}$, we have $c^{2}(t, \lambda)=z^{2}(t, \lambda)$ and hence $c(t, \lambda)= \pm z(t, \lambda)$. It follows that

$$
a(t, \lambda)= \pm x(t, \lambda) \text { and } b(t, \lambda)= \pm y(t, \lambda) .
$$

This contradicts the fact that $\gamma(t, \lambda) \cdot \nu(t, \lambda)=0$ for all $(t, \lambda) \in I \times \Lambda$. Hence $\widetilde{\nu}: I \times \Lambda \rightarrow S^{1}$ is a smooth mapping. By $\left(x_{t} a+y_{t} b+z_{t} c\right)(t, \lambda)=0$ and $\left(x_{t} x+y_{t} y+z_{t} z\right)(t, \lambda)=0$, we have

$$
\widetilde{\gamma}_{t}(t, \lambda) \cdot \widetilde{\nu}(t, \lambda)=0 \text { for all }(t, \lambda) \in I \times \Lambda .
$$

Therefore $(\widetilde{\gamma}, \widetilde{\nu}): I \times \Lambda \rightarrow D^{2} \times S^{1}$ is a one-parameter family of Legendre curves. By a similar calculation as in Proposition 4.1, we have the curvature $(\widetilde{\ell}, \widetilde{m}, \widetilde{\beta})$ of $(\widetilde{\gamma}, \widetilde{\nu})$.

Proposition 4.6. Under the same assumptions in Proposition 4.5, suppose that $e: U \rightarrow I \times \Lambda$ is a pre-envelope of $(\gamma, \nu)$ and $E_{\gamma}: U \rightarrow S^{2}$ is an envelope. Then $e: U \rightarrow I \times \Lambda$ is also a pre-envelope of $(\widetilde{\gamma}, \widetilde{\nu}): I \times \Lambda \rightarrow D^{2} \times S^{1}$. Moreover, we have $E_{\widetilde{\gamma}}(u)=\widetilde{E}_{\gamma}(u)$ for all $u \in U$, where $E_{\widetilde{\gamma}}=\widetilde{\gamma} \circ$ e and $\widetilde{E}_{\gamma}=\pi \circ E_{\gamma}$.

Proof. Since $e: U \rightarrow I \times \Lambda$ is a pre-envelope of $(\gamma, \nu)$, we have $\gamma_{\lambda}(e(u)) \cdot \nu(e(u))=0$ for all $u \in U$. It follows that

$$
\left(x_{\lambda}(t, \lambda)(a(t, \lambda) z(t, \lambda)-x(t, \lambda) c(t, \lambda))+y_{\lambda}(t, \lambda)(b(t, \lambda) z(t, \lambda)-y(t, \lambda) c(t, \lambda))\right) \circ e(u)=0 .
$$

By a direct calculation, we have $\widetilde{\gamma}_{\lambda}(e(u)) \cdot \widetilde{\nu}(e(u))=0$ for all $u \in U$. Therefore $e: U \rightarrow I \times \Lambda$ is a pre-envelope of $(\widetilde{\gamma}, \widetilde{\nu})\left(\right.$ cf. [11]). Moreover, we have $E_{\widetilde{\gamma}}(u)=\widetilde{\gamma} \circ e(u)=\pi \circ \gamma \circ e(u)=\pi\left(E_{\gamma}(u)\right)=\widetilde{E}_{\gamma}(u)$ for all $u \in U$. 
Conversely, we have the following results.

Proposition 4.7. Let $(\widetilde{\gamma}, \widetilde{\nu}): I \times \Lambda \rightarrow D^{2} \times S^{1}$ be a one-parameter family of Legendre curves with the curvature $(\widetilde{\ell}, \widetilde{m}, \widetilde{\beta})$. We denote

$$
\widetilde{\gamma}(t, \lambda)=(x(t, \lambda), y(t, \lambda)), \widetilde{\nu}(t, \lambda)=(a(t, \lambda), b(t, \lambda)) .
$$

Then we have $(\gamma, \nu): I \times \Lambda \rightarrow \Delta \subset S^{+} \times S^{2}$ is a one-parameter family of Legendre curves, where

$$
\begin{aligned}
& \gamma(t, \lambda)=\pi^{-1} \circ \widetilde{\gamma}(t, \lambda)=(x(t, \lambda), y(t, \lambda) z(t, \lambda)), \\
& \nu(t, \lambda)=\frac{1}{\sqrt{1-(x a+y b)^{2}}}(a-x(x a-y b), b-y(x a-y b),-z(x a+y b))(t, \lambda) .
\end{aligned}
$$

Here we put $z(t, \lambda)=\sqrt{1-x(t, \lambda)^{2}-y(t, \lambda)^{2}}$.

Proof. Since $\widetilde{\gamma}(t, \lambda) \cdot \widetilde{\gamma}(t, \lambda)<1$ and $\widetilde{\nu}(t, \lambda) \cdot \widetilde{\nu}(t, \lambda)=1$, we have $x(t, \lambda) a(t, \lambda)+y(t, \lambda) b(t, \lambda)<1$ for all $(t, \lambda) \in I \times \Lambda$. Therefore $\nu: I \times \Lambda \rightarrow S^{2}$ is a smooth mapping. By the same argument as in Proposition 4.5, we have

$$
\begin{aligned}
& \widetilde{\ell}(t, \lambda)=-a_{t}(t, \lambda) b(t, \lambda)+a(t, \lambda) b_{t}(t, \lambda), \\
& \widetilde{\beta}(t, \lambda)=-x_{t}(t, \lambda) b(t, \lambda)+y_{t}(t, \lambda) a(t, \lambda), \\
& \widetilde{m}(t, \lambda)=-a_{\lambda}(t, \lambda) b(t, \lambda)+a(t, \lambda) b_{\lambda}(t, \lambda) .
\end{aligned}
$$

Since

$$
\gamma_{t}(t, \lambda)=\left(x_{t}(t, \lambda), y_{t}(t, \lambda),-\frac{x(t, \lambda) x_{t}(t, \lambda)-y(t, \lambda) y_{t}(t, \lambda)}{z(t, \lambda)}\right), x^{2}(t, \lambda)+y^{2}(t, \lambda)+z^{2}(t, \lambda)=1,
$$

we have $\gamma(t, \lambda) \cdot \nu(t, \lambda)=0$ and $\gamma_{t}(t, \lambda) \cdot \nu(t, \lambda)=0$ for all $(t, \lambda) \in I \times \Lambda$. Therefore $(\gamma, \nu): I \times \Lambda \rightarrow \Delta \subset S^{+} \times S^{2}$ is a one-parameter family of Legendre curves.

Remark 4.8. We can detect to the curvature $(m, n, L, M, N)$ of $(\gamma, \nu)$ in Proposition 4.7. However, the description is a little bit long, we omit it.

Proposition 4.9. Under the same assumptions in Proposition 4.7, suppose that $e: U \rightarrow I \times \Lambda$ is a pre-envelope of $(\widetilde{\gamma}, \widetilde{\nu})$ and $E_{\widetilde{\gamma}}: U \rightarrow \mathbb{R}^{2}$ is an envelope. Then $e: U \rightarrow I \times \Lambda$ is also a pre-envelope of $(\gamma, \nu): I \times \Lambda \rightarrow \Delta \subset S^{+} \times S^{2}$. Moreover, we have $\pi^{-1} \circ E_{\widetilde{\gamma}}(u)=E_{\gamma}(u)$ for all $u \in U$.

Proof. Since $e: U \rightarrow I \times \Lambda$ is a pre-envelope of $(\widetilde{\gamma}, \widetilde{\nu})$, we have $\widetilde{\gamma}_{\lambda}(e(u)) \cdot \widetilde{\nu}(e(u))=0$ for all $u \in U$ (cf. [11]). It follows that

$$
\left(x_{\lambda}(t, \lambda) \cdot a(t, \lambda)+y_{\lambda}(t, \lambda) \cdot b(t, \lambda)\right) \circ e(u)=0 .
$$

By a direct calculation, we have $\gamma_{\lambda}(e(u)) \cdot \nu(e(u))=0$ for all $u \in U$. By Theorem 3.3, $e: U \rightarrow I \times \Lambda$ is a pre-envelope of $(\gamma, \nu)$. Moreover, we have $\pi^{-1} \circ E_{\widetilde{\gamma}}(u)=\pi^{-1} \circ \widetilde{\gamma} \circ e(u)=\gamma \circ e(u)=E_{\gamma}(u)$ for all $u \in U$.

\section{Examples}

Example 5.1. Let $(\gamma, \nu):[0,2 \pi) \times[0,2 \pi) \rightarrow \Delta$,

$$
\begin{aligned}
& \gamma(t, \theta)=\left(\cos \theta\left(\frac{3}{4} \cos t-\frac{1}{4} \cos 3 t\right)-\frac{\sqrt{3}}{2} \sin \theta \cos t, \frac{3}{4} \sin t-\frac{1}{4} \sin 3 t, \frac{\sqrt{3}}{2} \cos \theta \cos t+\sin \theta\left(\frac{3}{4} \cos t-\frac{1}{4} \cos 3 t\right)\right) \\
& \nu(t, \theta)=\left(\cos \theta\left(-\frac{3}{4} \sin t-\frac{1}{4} \sin 3 t\right)-\frac{\sqrt{3}}{2} \sin \theta \sin t, \frac{3}{4} \cos t+\frac{1}{4} \cos 3 t, \frac{\sqrt{3}}{2} \cos \theta \sin t-\sin \theta\left(\frac{3}{4} \sin t+\frac{1}{4} \sin 3 t\right)\right) .
\end{aligned}
$$

Then $(\gamma, \nu)$ is a one-parameter family of Legendre curves. By definition

$$
\begin{aligned}
\boldsymbol{\mu}(t, \theta)= & \gamma(t, \theta) \times \nu(t, \theta) \\
= & \left(-\frac{3 \sqrt{3}}{4} \cos \theta \cos ^{2} t-\frac{\sqrt{3}}{8} \cos \theta \cos 2 t+\frac{3 \sqrt{3}}{8} \cos \theta-\frac{1}{2} \sin \theta,-\sqrt{3} \sin t \cos t,\right. \\
& \left.-\frac{3 \sqrt{3}}{4} \sin \theta \cos ^{2} t-\frac{\sqrt{3}}{8} \sin \theta \cos 2 t+\frac{3 \sqrt{3}}{8} \sin \theta+\frac{1}{2} \cos \theta\right) .
\end{aligned}
$$


Then the curvature is given by

$$
\begin{aligned}
m(t, \theta) & =\frac{\sqrt{3}}{32}\left(96 \sin t \cos ^{4} t-54 \sin t \cos ^{2} t+9 \sin t \cos t \cos 3 t-33 \sin 3 t \cos ^{2} t-20 \sin t+12 \sin 3 t\right) \\
n(t, \theta) & =\frac{\sqrt{3}}{8}(6 \cos t \cos 2 t+5 \cos t-3 \cos 3 t), \\
L(t, \theta) & =\sqrt{3} \sin t \cos t, M(t, \theta)=\frac{9}{8}\left(2 \cos ^{2} t-1\right)-\frac{1}{8} \cos 3 t, N(t, \theta)=\frac{3}{4} \sin t \cos ^{2} t+\frac{1}{16} \sin 3 t-\frac{15}{16} \sin t .
\end{aligned}
$$

If we take

$$
\begin{aligned}
& e:[0,2 \pi) \rightarrow[0,2 \pi) \times[0,2 \pi), \\
& e(u)=(0, u),(\pi / 2, u),(\pi, u),(3 \pi / 2, u),
\end{aligned}
$$

then we got $L(e(u))=0$ for all $u \in[0,2 \pi)$. Thus, e are pre-envelopes of $(\gamma, \nu)$. Hence, the envelopes $E_{\gamma}:[0,2 \pi) \rightarrow S^{2}$ are given by

$$
\begin{aligned}
& E_{\gamma}=(1 / 2 \cos u-\sqrt{3} / 2 \sin u, 0,1 / 2 \sin u+\sqrt{3} / 2 \cos u),(0,1,0), \\
& (-1 / 2 \cos u+\sqrt{3} / 2 \sin u, 0,-1 / 2 \sin u-\sqrt{3} / 2 \cos u),(0,-1,0),
\end{aligned}
$$

see Figure 1.

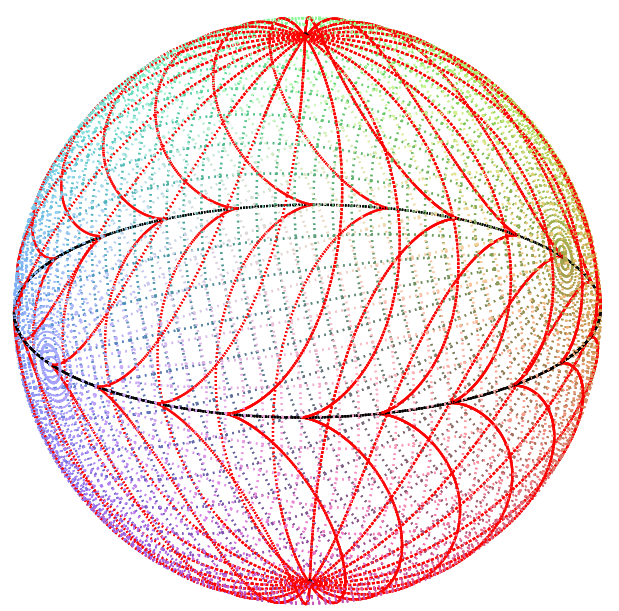

Figure 1: The red curves are one-parameter family of Legendre curves $(\gamma, \nu)$. The black curve and the two black points are the envelopes $E_{\gamma}$.

Example 5.2. Let $n, m$ and $k$ be natural numbers with $m=k+n$. We give a mapping $(\gamma, \nu): \mathbb{R} \times[0,2 \pi) \rightarrow \Delta$ by

$$
\begin{aligned}
& \gamma(t, \theta)=\frac{1}{\sqrt{t^{2 m}+t^{2 n}+1}}\left(\cos \theta-t^{n} \sin \theta, \sin \theta+t^{n} \cos \theta, t^{m}\right), \\
& \nu(t, \theta)=\frac{1}{\sqrt{k^{2} t^{2 m}+m^{2} t^{2 k}+n^{2}}}\left(k t^{m} \cos \theta+m t^{k} \sin \theta, k t^{m} \sin \theta-m t^{k} \cos \theta, n\right) .
\end{aligned}
$$

Then $(\gamma, \nu): \mathbb{R} \times[0,2 \pi) \rightarrow \Delta$ is a one-parameter family of Legendre curves. By definition,

$$
\begin{aligned}
\boldsymbol{\mu}(t, \theta)= & \gamma(t, \theta) \times \nu(t, \theta) \\
= & \frac{1}{\sqrt{t^{2 m}+t^{2 n}+1} \sqrt{k^{2} t^{2 m}+m^{2} t^{2 k}+n^{2}}}\left(t^{n}\left(m t^{2 k}+n\right) \cos \theta+\left(-k t^{2 m}+n\right) \sin \theta,\right. \\
& \left.t^{n}\left(m t^{2 k}+n\right) \sin \theta+\left(k t^{2 m}-n\right) \cos \theta,-k t^{2 n+k}-m t^{k}\right) .
\end{aligned}
$$


Then the curvature is given by

$m(t, \theta)=-\frac{t^{n-1} \sqrt{k^{2} t^{2 m}+m^{2} t^{2 k}+n^{2}}}{t^{2 m}+t^{2 n}+1}, n(t, \theta)=\frac{m n k t^{k-1} \sqrt{t^{2 m}+t^{2 n}+1}}{k^{2} t^{2 m}+m^{2} t^{2 k}+n^{2}}$,
$L(t, \theta)=-\frac{m t^{k}+k t^{m+n}}{\sqrt{t^{2 m}+t^{2 n}+1} \sqrt{k^{2} t^{2 m}+m^{2} t^{2 k}+n^{2}}}, M(t, \theta)=\frac{-n}{\sqrt{k^{2} t^{2 m}+m^{2} t^{2 k}+n^{2}}}, N(t, \theta)=\frac{t^{m}}{\sqrt{t^{2 m}+t^{2 n}+1}}$.

If we take $e:[0,2 \pi) \rightarrow \mathbb{R} \times[0,2 \pi), e(u)=(0, u)$, then we got $L(e(u))=0$ for all $u \in[0,2 \pi)$. Thus, e is a pre-envelope of $(\gamma, \nu)$. Hence, the envelope $E_{\gamma}:[0,2 \pi) \rightarrow S^{2}$ is given by $E_{\gamma}(u)=(\cos u, \sin u, 0)$.

For example, when $n=2, m=3, k=1$, then we have

$$
\begin{aligned}
& \gamma(t, \theta)=\frac{1}{\sqrt{t^{6}+t^{4}+1}}\left(\cos \theta-t^{2} \sin \theta, \sin \theta+t^{2} \cos \theta, t^{3}\right), \\
& \nu(t, \theta)=\frac{1}{\sqrt{t^{6}+9 t^{2}+4}}\left(t^{3} \cos \theta+3 t \sin \theta, t^{3} \sin \theta-3 t \cos \theta, 2\right) .
\end{aligned}
$$

By definition,

$$
\begin{aligned}
\boldsymbol{\mu}(t, \theta)= & \gamma(t, \theta) \times \nu(t, \theta) \\
= & \frac{1}{\sqrt{t^{6}+t^{4}+1} \sqrt{t^{6}+9 t^{2}+4}}\left(t^{2}\left(3 t^{2}+2\right) \cos \theta+\left(-t^{6}+2\right) \sin \theta, t^{2}\left(3 t^{2}+2\right) \sin \theta+\left(t^{6}-2\right) \cos \theta,\right. \\
& \left.-t^{5}-3 t\right) .
\end{aligned}
$$

Then the curvature is given by

$$
\begin{aligned}
& m(t, \theta)=\frac{-t \sqrt{t^{6}+9 t^{2}+4}}{t^{6}+t^{4}+1}, \quad n(t, \theta)=\frac{6 \sqrt{t^{6}+t^{4}+1}}{t^{6}+9 t^{2}+4} \\
& L(t, \theta)=\frac{-t^{5}-3 t}{\sqrt{t^{6}+9 t^{2}+4} \sqrt{t^{6}+t^{4}+1}}, \quad M(t, \theta)=\frac{-2}{\sqrt{t^{6}+9 t^{2}+4}}, \quad N(t, \theta)=\frac{t^{3}}{\sqrt{t^{6}+t^{4}+1}} .
\end{aligned}
$$

The envelope $E_{\gamma}:[0,2 \pi) \rightarrow S^{2}$ is given by $E_{\gamma}(u)=(\cos u, \sin u, 0)$, see Figure 2. The parallel curve of the envelope $E_{\gamma}(u)$ for $\lambda=\pi / 6$ is $E_{\gamma}^{\frac{\pi}{6}}(u)=(\sqrt{3} / 2 \cos u, \sqrt{3} / 2 \sin u,-1 / 2)$.

The parallel curves of $(\gamma, \nu)$ is

$$
\begin{aligned}
\gamma^{\frac{\pi}{6}}(t, \theta)= & \cos \frac{\pi}{6} \gamma(t, \theta)-\sin \frac{\pi}{6} \nu(t, \theta) \\
= & \left(\frac{\sqrt{3}\left(\cos \theta-t^{2} \sin \theta\right)}{2 \sqrt{t^{6}+t^{4}+1}}-\frac{t^{3} \cos \theta+3 t \sin \theta}{2 \sqrt{t^{6}+9 t^{2}+4}}, \frac{\sqrt{3}\left(\sin \theta+t^{2} \cos \theta\right)}{2 \sqrt{t^{6}+t^{4}+1}}-\frac{t^{3} \sin \theta-3 t \cos \theta}{2 \sqrt{t^{6}+9 t^{2}+4}},\right. \\
& \left.\frac{\sqrt{3} t^{3}}{2 \sqrt{t^{6}+t^{4}+1}}-\frac{1}{\sqrt{t^{6}+9 t^{2}+4}}\right) .
\end{aligned}
$$

Then the envelope of these parallel curves is given by $E_{\gamma^{\frac{\pi}{6}}}(u)=(\sqrt{3} / 2 \cos u, \sqrt{3} / 2 \sin u,-1 / 2)$. Thus, we have $E_{\gamma}^{\frac{\pi}{6}}(u)=E_{\gamma \frac{\pi}{6}}(u)$ for all $u \in U$. Figure 3 put these curves in the one sphere.

\section{CONFLICT OF INTERESTS}

The authors declare that there is no conflicts of interests in this work.

\section{Acknowledgement}

The authors would like to thank the referee for helpful comments to improve the original manuscript. This work was supported by the National Natural Science Foundation of China (Grant No. 11671070), China Scholarship Council (Grant No. 201606620072) and JSPS KAKENHI (Grant No. JP 17K05238).

\section{References}

[1] V. I. Arnol'd, Singularities of Caustics and Wave Fronts. Mathematics and Its Applications 62 Kluwer Academic Publishers (1990).

[2] V. I. Arnol'd, S. M. Gusein-Zade and A. N. Varchenko, Singularities of Differentiable Maps. (1986).

[3] J. W. Bruce and P. J. Giblin, What is an envelope? Math. Gaz. 65 (1981), 186-192. 


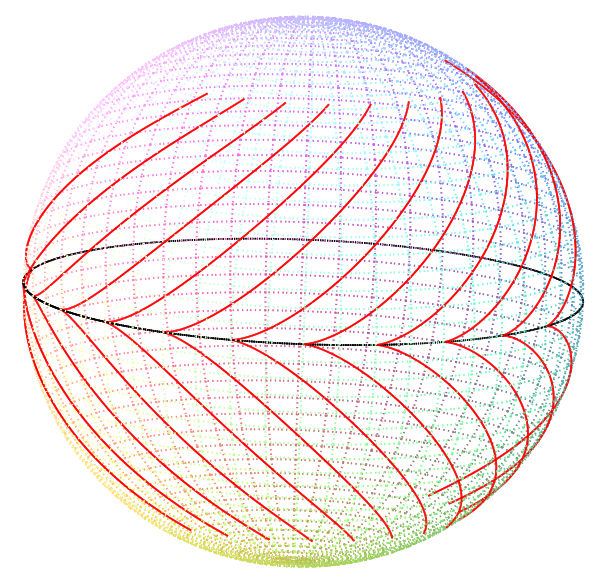

Figure 2: The red curves are one-parameter family of Legendre curves $(\gamma, \nu)$. The black curve is their envelope $E_{\gamma}$.

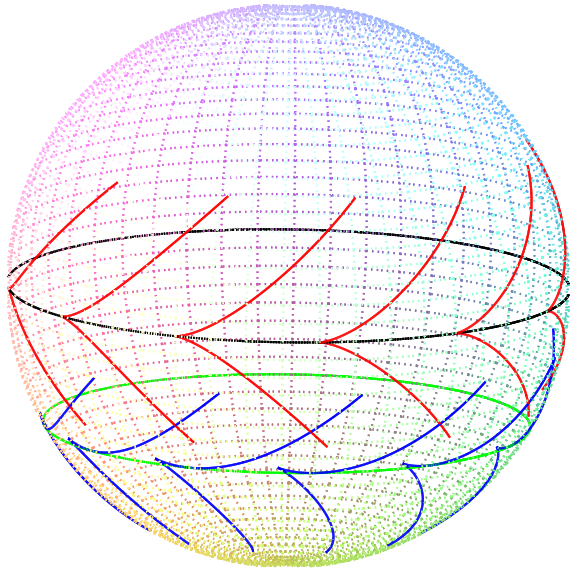

Figure 3: The red curves are one-parameter family of Legendre curves $(\gamma, \nu)$. The black curve is their envelope $E_{\gamma}$. The blue curves are their parallel curves. The green curve is the envelope of these parallel curves.

[4] J. W. Bruce and P. J. Giblin, Curves and Singularities. A geometrical introduction to singularity theory. Second edition. Cambridge University Press, Cambridge, 1992.

[5] C. G. Gibson, Elementary Geometry of Differentiable Curves. An undergraduate introduction. Cambridge University Press, Cambridge, 2001.

[6] A. Gray, E. Abbena, and S. Salamon, Modern differential geometry of curves and surfaces with Mathematica. Third edition. Studies in Advanced Mathematics. Chapman and Hall/CRC, Boca Raton, FL, 2006

[7] G. Ishikawa, Singularities of Curves and Surfaces in Various Geometric Problems. CAS Lecture Notes 10, Exact Sciences. 2015.

[8] S. Izumiya, M. C. Romero-Fuster, M. A. S. Ruas, F. Tari, Differential Geometry from a Singularity Theory Viewpoint. World Scientific Pub. Co Inc. 2015

[9] J. W. Rutter, Geometry of Curves. Chapman \& Hall/CRC Mathematics. Chapman \& Hall/CRC, Boca Raton, FL, 2000.

[10] M. Takahashi, Legendre curves in the unit spherical bundle over the unit sphere and evolutes. Real and complex singularities, 337-355, Contemp. Math., 675, Amer. Math. Soc., Providence, RI, 2016.

[11] M. Takahashi, Envelopes of Legendre curves in the unit tangent bundle over the Euclidean plane. Results in Math. 71 (2017), 1473-1489. DOI:10.1007/s00025-016-0619-7. 